\title{
Convergence analysis of the nonconforming finite element discretization of Stokes and Navier-Stokes equations with nonlinear slip boundary conditions ${ }^{1}$
}

\author{
J. K. Djoko \\ Department of Mathematics and Applied Mathematics \\ University of Pretoria \\ 0002, South Africa \\ jules.djokokamdem@up.ac.za
}

\begin{abstract}
This work is concerned with the nonconforming finite approximations for the Stokes and Navier-Stokes equations driven by slip boundary condition of "friction" type. It is well documented that if the velocity is approximated by the Crouzeix-Raviart element of order one, while the discrete pressure is constant element wise the inequality of Korn doe not hold. Hence we propose a new formulation taking into account the curvature and the contribution of tangential velocity at the boundary. Using the maximal regularity of the weak solution, we derive a priori error estimates for the velocity and pressure by taking advantage of the enrichment mapping and the application of Babuska-Brezzi's theory for mixed problems.
\end{abstract}

Keywords: Stokes equations; Navier-Stokes equations; nonlinear slip boundary conditions; variational inequality; Crouzeix-Raviart element; a priori error estimate.

AMS subject classification: $65 \mathrm{~N} 30,76 \mathrm{D} 07,35 \mathrm{~J} 85$

\section{Introduction: Model Stokes and Navier-Stokes problems}

We consider steady flows of incompressible viscous fluid modeled by the Stokes system

$$
\begin{aligned}
& -2 \nu \operatorname{div} \boldsymbol{D}(\boldsymbol{u})+\nabla p \quad=\boldsymbol{f} \quad \text { in } \Omega, \\
& \operatorname{div} \boldsymbol{u}=0 \quad \text { in } \Omega,
\end{aligned}
$$

where $\Omega$, the flow region is a bounded domain in $\mathbb{R}^{2}$. The motion of the incompressible fluid is described by the velocity $\boldsymbol{u}(\boldsymbol{x})$ and pressure $p(\boldsymbol{x})$. In (1.1) $\boldsymbol{f}$ is the external body force per unit volume depending on $\boldsymbol{x}$, and $\nu$ is the positive parameter representing the kinematic viscosity. Equations (1.1) and (1.2) are supplemented by nonlinear slip boundary of friction type, which is the main modeling assumption in this work. It should be pointed out that such boundary conditions have already been considered in $[1,2,3,4,5,6,7]$. Hence we will just state the mathematical equations governing this phenomenon as the physical merit of such models have been discussed elsewhere (see particularly [6]). So, we assume that the boundary of $\Omega$, say, $\partial \Omega$ is made of two components $S$ and $\Gamma$, and it is required that $\partial \Omega=\overline{S \cup \Gamma}$, with $S \cap \Gamma=\emptyset$. Next, we consider the homogeneous Dirichlet condition on $\Gamma$, that is

$$
\boldsymbol{u}=0 \quad \text { on } \Gamma .
$$

\footnotetext{
${ }^{1}$ This work is dedicated to the memory of my late father Kamdem Sindjoun Joseph.
} 
We have chosen to work with a homogeneous condition on the velocity in order to avoid the technical arguments linked to the Hopf lemma (see [8], Chapter 4, Lemma 2.3). On $S$, we recall that the velocity is decomposed following its normal and tangential part as follows

$$
\boldsymbol{u}=u_{\boldsymbol{n}}+u_{\boldsymbol{\tau}}=(\boldsymbol{u} \cdot \boldsymbol{n}) \boldsymbol{n}+(\boldsymbol{u} \cdot \boldsymbol{\tau}) \boldsymbol{\tau},
$$

where $\boldsymbol{n}$ is the normal outward unit vector to $S$ and $\boldsymbol{\tau}$ is the tangent vector orthogonal to $\boldsymbol{n}$. On $S$, we first assume the impermeability condition

$$
\boldsymbol{u} \cdot \boldsymbol{n}=0 \text { on } S .
$$

In addition to (1.5), we also impose on $S$, a nonlinear slip boundary condition of friction type. But we first recall that the Cauchy stress tensor is $\boldsymbol{T}=-p \boldsymbol{I}+2 \nu \boldsymbol{D}(\boldsymbol{u})$, with the symmetric part of the velocity gradient $\boldsymbol{D}(\boldsymbol{u})=\frac{1}{2}\left[\nabla \boldsymbol{u}+(\nabla \boldsymbol{u})^{T}\right]$. Thus on $S$, the traction force $\boldsymbol{T} \boldsymbol{n}$ is decomposed as follows

$$
\begin{aligned}
\boldsymbol{T n} & =(\boldsymbol{T} \boldsymbol{n} \cdot \boldsymbol{n}) \boldsymbol{n}+(\boldsymbol{T} \boldsymbol{n} \cdot \boldsymbol{\tau}) \boldsymbol{\tau} \\
& =(-p+2 \nu \boldsymbol{n} \cdot \boldsymbol{D}(\boldsymbol{u}) \boldsymbol{n}) \boldsymbol{n}+2 \nu(\boldsymbol{\tau} \cdot \boldsymbol{D}(\boldsymbol{u}) \boldsymbol{n}) \boldsymbol{\tau} \\
& =(\boldsymbol{T} \boldsymbol{n})_{\boldsymbol{n}}+(\boldsymbol{T} \boldsymbol{n})_{\boldsymbol{\tau}} .
\end{aligned}
$$

Let $g: S \longrightarrow(0, \infty)$ be a non-negative function called threshold slip or barrier function, the nonlinear slip boundary conditions of friction type is formulated as follows (see $[1,2,3,4,5,6,7]$ )

$$
\left.\begin{array}{l}
\left|(\boldsymbol{T} \boldsymbol{n})_{\boldsymbol{\tau}}\right|<g \Rightarrow u_{\boldsymbol{\tau}}=\mathbf{0}, \\
\left|(\boldsymbol{T} \boldsymbol{n})_{\boldsymbol{\tau}}\right| \geq g \Rightarrow u_{\boldsymbol{\tau}} \neq \mathbf{0},-(\boldsymbol{T} \boldsymbol{n})_{\boldsymbol{\tau}}=g \frac{u_{\boldsymbol{\tau}}}{\left|u_{\boldsymbol{\tau}}\right|}
\end{array}\right\} \text { on } S,
$$

where $|\boldsymbol{v}|^{2}=\boldsymbol{v} \cdot \boldsymbol{v}$ is the Euclidean norm. Equation (1.7) expresses the fact that $(\boldsymbol{T n})_{\boldsymbol{\tau}}$ and $\boldsymbol{u}_{\boldsymbol{\tau}}$ are parallel but opposite. On the other hand (1.7) is equivalent to (see [9])

$$
-(\boldsymbol{T n})_{\boldsymbol{\tau}} \in g \partial\left|u_{\boldsymbol{\tau}}\right| \quad \text { on } S,
$$

where $\partial|\cdot|$ is the sub-differential of the real-valued function $|\cdot|$.

We recall that if $\mathcal{X}$ is a Hilbert space with $x_{0} \in \mathcal{X}$, and $y \in \mathcal{X}^{\prime}$, then

$$
y \in \partial \Psi\left(x_{0}\right) \text { means that } \Psi(x)-\Psi\left(x_{0}\right) \geq y \cdot\left(x-x_{0}\right) \quad \forall x \in \mathcal{X} .
$$

We will refer to boundary-value problem (1.1)-(1.7) as problem (P).

The Stokes system can be considered as a simplification of the Navier-Stokes system of equations, where (1.2) is replaced by

$$
-2 \nu \operatorname{div} \boldsymbol{D}(\boldsymbol{u})+(\boldsymbol{u} \cdot \nabla) \boldsymbol{u}+\nabla p=\boldsymbol{f} \quad \text { in } \quad \Omega,
$$

and (1.2), (1.3), (1.5) and (1.7) are unchanged. Here,

$$
(\boldsymbol{u} \cdot \nabla) \boldsymbol{u}=\sum_{i=1}^{2} u_{i} \frac{\partial \boldsymbol{u}}{\partial x_{i}}
$$

is the convection term. We will refer to boundary-value problem (1.2), (1.3), (1.5), (1.7) and (1.10), as problem (F).

The main concern in this research is to analyze numerically problem $(\mathbf{P})$ and problem $(\mathbf{F})$ using the nonconforming finite element method where the velocity is approximated by lowest order 
Crouzeix-Raviart element and the pressure with piecewise constant functions [10]. The a priori error analysis of problem (P) has been proposed with discontinuous Galerkin method in [11], while numerous studies using conforming approximation of the velocity have been contributed by researchers, see among others $[12,13,14,15,16,17,18]$.

The finite element method is now well adapted for approximating the solution of partial differential equations written in weak form (including variational inequalities, see [19, 20, 21, 22, 23, 24]), and the search for efficient and simple non conforming finite element methods for Stokes, NavierStokes equations driven by nonlinear slip boundary conditions has not yet been well explored by researchers (except the early work of the author in [11]). At this point we should mention that some of the major difficulties associated with problem $(\mathbf{P})$ and problem $(\mathbf{F})$ include; the incompressibility condition and the related unknown pressure, the nonlinear slip boundary condition, and the convection term for Navier-Stokes equations. It should be observed that for both problems, the nonlinear slip boundary condition (1.7) is responsible for the inequality relation appearing in the variational formulation, while the velocity $\boldsymbol{u}$ is related to the pressure via the incompressibility condition, $\operatorname{div} \boldsymbol{u}=0$. Thus the pressure is viewed as a Lagrange multiplier. Hence, both problems can be formulated as a mixed variational problem, which can be shown to be equivalent to many other variational problems $[6,7]$.

Recently, so much works have been done in the finite element community using discontinuous Galerkin methods and nonconforming approaches, and comparison have been established with the classical conforming approach [25]. It turns out that it is simpler to implement nonconforming approaches (probably because the basis functions have smaller support compared to the conforming approximations), and the proof of inf-sup condition for mixed formulation is simpler when nonconforming methods are used. Hence, this work can be viewed as the continuation of a work started in [11], in the sense that we show that Crouzeix-Raviart's element can be used to approximate successfully the velocity of the Stokes problems driven by nonlinear slip boundary conditions.

The mixed finite element approximation for variational inequalities presented in [23, 24] are motivated by problems in plasticity, while the analysis in [12] uses the penalty approach in the Stokes equations to circumvent the incompressibility constraint. Using a different type of slip boundary condition R. Verfurth [26] has analyzed the problem by relaxing the constraint (1.5) at the expense of an additional unknown. In [22] a solution technique and the convergence of an algorithm for solving the Stokes equations with leak and slip boundary conditions is presented, but the mathematical analysis of the finite element method presented is not discussed. Our framework for analyzing the finite element discretizations of problem $(\mathbf{P})$ and problem $(\mathbf{F})$ is based on a suitable extension of the mixed finite theory of Babuška-Brezzi [27], reminiscent of those used in, e.g., [23, 24] for the analysis of problems in plasticity. We formulate and analyze the non conforming finite element approximations associated to problem $(\mathbf{P})$ and problem $(\mathbf{F})$ without penalization by considering the mixed variational approach in which the velocity and pressure satisfy the Babuška-Brezzi (BB) condition [27]. The non conforming finite elements approximations are constructed on a regular decomposition of the domain [28].

The rest of our work is organised follows. In Section 2, we reformulate problem (P) and problem (F) in terms of variational inequalities and indicate how their solvability are obtained. We also introduce some notations pertaining to the nonconforming approximations and formulate the discrete problems based on Crouziex-Raviart's approximations. In Section 3, we discuss the a priori error related to problem $(\mathbf{P})$, while in Section 4, we discuss the a priori error associated with problem (F). Some conclusions and future research are drawn in Section 5 . 


\section{Nonlinear slip boundary conditions/ Nonconforming fi- nite element approximations}

This section introduces notation on variational inequality. We also formulate two finite element approximations

\subsection{Notation/preliminaries}

We adopt the standard definitions [29] for the Sobolev spaces $H^{s}(D)$ and their associated inner products $(\cdot, \cdot)_{s, D}$, norms $\|\cdot\|_{s, D}$, and semi-norms $|\cdot|_{s, D}$ for $s \geq 0$. The space $H^{0}(D)$ coincides with $L^{2}(D)$, for which the norm and inner product are denoted as $\|\cdot\|_{D}$ and $(\cdot, \cdot)_{D}$, respectively. If $D=\Omega$, we drop $D$.

Throughout this work, boldface characters denote vector quantities, and $\boldsymbol{H}^{1}(\Omega)=H^{1}(\Omega)^{2}$ and $\boldsymbol{L}^{2}(\Omega)=L^{2}(\Omega)^{2}$. The following functional spaces will be helpful in the analysis of various weak formulations that we will introduce later.

$$
\begin{aligned}
\boldsymbol{V} & =\left\{\boldsymbol{v} \in \boldsymbol{H}^{1}(\Omega):\left.\quad \boldsymbol{v}\right|_{\Gamma}=0,\left.\boldsymbol{v} \cdot \boldsymbol{n}\right|_{S}=0\right\}, \\
M & =L_{0}^{2}=\left\{p \in L^{2}(\Omega): \quad(p, 1)=0\right\}, \\
\boldsymbol{V}_{\mathrm{div}} & =\left\{\boldsymbol{v} \in \boldsymbol{H}^{1}(\Omega):\left.\operatorname{div} \boldsymbol{v}\right|_{\Omega}=0,\left.\quad \boldsymbol{v}\right|_{\Gamma}=0,\left.\boldsymbol{v} \cdot \boldsymbol{n}\right|_{S}=0\right\} .
\end{aligned}
$$

It can be shown at least formally that problem (1.1)-(1.7) is equivalent to:

$$
\left\{\begin{array}{l}
\text { Find } \boldsymbol{u} \in \boldsymbol{V}_{\mathrm{div}}, \text { such that for all } \boldsymbol{v} \in \boldsymbol{V}_{\mathrm{div}}, \\
2 \nu(\boldsymbol{D}(\boldsymbol{u}), \boldsymbol{D}(\boldsymbol{v}-\boldsymbol{u}))+\left(g,\left|\boldsymbol{v}_{\boldsymbol{\tau}}\right|-\left|\boldsymbol{u}_{\boldsymbol{\tau}}\right|\right)_{S} \geq(\boldsymbol{f}, \boldsymbol{v}-\boldsymbol{u}) .
\end{array}\right.
$$

It is well documented in the literature (see $[30,31]$ ) that if the velocity is approximated with $\mathcal{P}_{1}$ non-conforming element, the quadratic form $(\boldsymbol{D}(\boldsymbol{v}), \boldsymbol{D}(\boldsymbol{v}))$ is not positive definite. Hence, an alternative formulation is needed. One approach to overcome the difficulty highlighted is to proceed as in discontinuous Galerkin discretization, which amounts to add stabilization like terms (see [11, 32]). In [11], symmetric and non-symmetric interior penalty Galerkin approaches are considered and convergence is demonstrated. Whereas in [32] the over stabilized formulation is analyzed. In this work we want to take advantage of the decomposition of $(\boldsymbol{T n})_{\boldsymbol{\tau}}=2 \nu(\boldsymbol{\tau} \cdot \boldsymbol{D}(\boldsymbol{u}) \boldsymbol{n}) \boldsymbol{\tau}$ on $S$ and propose a new formulation based on the gradient of the velocity field. For that purpose, we need to introduce some notations to describe the boundary conditions on $S$. Let $\boldsymbol{\psi}: S \rightarrow \mathbb{R}^{2}$ be a regular curve parameterized by its arc length $s$. The functions $x(s), y(s)$ are assumed to be in $\mathcal{C}^{2}(S)$. Here and henceforth, a superscript prime denotes the derivative with respect to $s$. The function $\left(x^{\prime}(s), y^{\prime}(s)\right)=\boldsymbol{\psi}^{\prime}(s)$ is called the tangent vector of the curve $\boldsymbol{\psi}$ at $s$, and denoted by $\boldsymbol{\tau}$. It is assumed that the length of $\tau$ is one. Since the curve is assumed to be regular, the tangent vector is defined at each point along the curve. The length of $\boldsymbol{\psi}^{\prime \prime}(s)$ is called principal curvature of $\boldsymbol{\psi}$ at $s$ and denoted by $\kappa(s)$. A unit vector $\boldsymbol{n}$ in the direction of $\boldsymbol{\psi}^{\prime \prime}(s)$ is taken by requiring it to form with the tangent vector $\boldsymbol{t}$ an orthonormal basis at each point on the curve, and to satisfy the equation

$$
\boldsymbol{\tau}^{\prime}=\boldsymbol{\psi}^{\prime \prime}(s)=\kappa(s) \boldsymbol{n} .
$$

Conventionally, we choose the direction $\boldsymbol{n}$ towards the convex side of the curve $\psi$ at $s$. Moreover, by differentiating the equation $\boldsymbol{\tau} \cdot \boldsymbol{\tau}=1$, one obtains $\boldsymbol{\tau}^{\prime} \cdot \boldsymbol{\tau}=0$, so that $\psi^{\prime \prime}(s)$ is normal to $\boldsymbol{\psi}^{\prime}(s)$, that is $\boldsymbol{n} \cdot \boldsymbol{\tau}=0$. The vector $\boldsymbol{n}$ is called the normal vector at $s$. In this work, we need a relation 
between $(\boldsymbol{T} \boldsymbol{n})_{\boldsymbol{\tau}}$ and $\boldsymbol{u}_{\boldsymbol{\tau}}$. For that purpose we follow P. Grisvard [33] (Chapter 3). We consider a smooth vector field $\boldsymbol{v}$ so that $\boldsymbol{v}$ and $\nabla \boldsymbol{v}$ are defined on $S$. With the vectors $\boldsymbol{n}$ and $\boldsymbol{\tau}$ introduced above, one has the following decomposition on $S$

$$
\boldsymbol{v}=v_{\boldsymbol{n}}+v_{\boldsymbol{\tau}}=(\boldsymbol{v} \cdot \boldsymbol{n}) \boldsymbol{n}+(\boldsymbol{v} \cdot \boldsymbol{\tau}) \boldsymbol{\tau}
$$

In what follows $\partial / \partial \boldsymbol{n}$, denotes differentiation in the direction of $\boldsymbol{n}$. The gradient of $\boldsymbol{v}$ on $S$ is then given by

$$
\begin{aligned}
\nabla \boldsymbol{v}= & \frac{\partial \boldsymbol{v}}{\partial s} \boldsymbol{\tau}^{T}+\frac{\partial \boldsymbol{v}}{\partial \boldsymbol{n}} \boldsymbol{n}^{T} \\
= & \frac{\partial}{\partial s}((\boldsymbol{v} \cdot \boldsymbol{n}) \boldsymbol{n}+(\boldsymbol{v} \cdot \boldsymbol{\tau}) \boldsymbol{\tau}) \boldsymbol{\tau}^{T}+\frac{\partial}{\partial \boldsymbol{n}}((\boldsymbol{v} \cdot \boldsymbol{n}) \boldsymbol{n}+(\boldsymbol{v} \cdot \boldsymbol{\tau}) \boldsymbol{\tau}) \boldsymbol{n}^{T} \\
= & \left(\frac{\partial(\boldsymbol{v} \cdot \boldsymbol{n})}{\partial s} \boldsymbol{n}+(\boldsymbol{v} \cdot \boldsymbol{n}) \frac{\partial \boldsymbol{n}}{\partial s}+\frac{\partial(\boldsymbol{v} \cdot \boldsymbol{\tau})}{\partial s} \boldsymbol{\tau}+(\boldsymbol{v} \cdot \boldsymbol{\tau}) \frac{\partial \boldsymbol{\tau}}{\partial s}\right) \boldsymbol{\tau}^{T} \\
& +\left(\frac{\partial(\boldsymbol{v} \cdot \boldsymbol{n})}{\partial \boldsymbol{n}} \boldsymbol{n}+(\boldsymbol{v} \cdot \boldsymbol{n}) \frac{\partial \boldsymbol{n}}{\partial \boldsymbol{n}}+\frac{\partial(\boldsymbol{v} \cdot \boldsymbol{\tau})}{\partial \boldsymbol{n}}+(\boldsymbol{v} \cdot \boldsymbol{\tau}) \frac{\partial \boldsymbol{\tau}}{\partial \boldsymbol{n}}\right) \boldsymbol{n}^{T}
\end{aligned}
$$

Hence from $\boldsymbol{n} \boldsymbol{\tau}^{T}=0, \boldsymbol{n} \boldsymbol{n}^{T}=\boldsymbol{\tau} \boldsymbol{\tau}^{T}=1$, and $\frac{\partial \boldsymbol{\tau}}{\partial \boldsymbol{n}} \cdot \boldsymbol{\tau}=0$ one obtains

$$
\begin{aligned}
(\nabla \boldsymbol{v}) \boldsymbol{n} & =\frac{\partial(\boldsymbol{v} \cdot \boldsymbol{n})}{\partial \boldsymbol{n}}+(\boldsymbol{v} \cdot \boldsymbol{n}) \frac{\partial \boldsymbol{n}}{\partial \boldsymbol{n}}+\frac{\partial(\boldsymbol{v} \cdot \boldsymbol{\tau})}{\partial \boldsymbol{n}} \boldsymbol{\tau}+(\boldsymbol{v} \cdot \boldsymbol{\tau}) \frac{\partial \boldsymbol{\tau}}{\partial \boldsymbol{n}} \\
\boldsymbol{\tau} \cdot(\nabla \boldsymbol{v}) \boldsymbol{n} & =(\boldsymbol{v} \cdot \boldsymbol{n})\left(\frac{\partial \boldsymbol{n}}{\partial \boldsymbol{n}}\right) \cdot \boldsymbol{\tau}+\frac{\partial(\boldsymbol{v} \cdot \boldsymbol{\tau})}{\partial \boldsymbol{n}}
\end{aligned}
$$

Next, from (2.4) one has

$$
\begin{aligned}
(\nabla \boldsymbol{v})^{T}= & \boldsymbol{\tau}\left(\frac{\partial(\boldsymbol{v} \cdot \boldsymbol{n})}{\partial s} \boldsymbol{n}^{T}+(\boldsymbol{v} \cdot \boldsymbol{n})\left(\frac{\partial \boldsymbol{n}}{\partial s}\right)^{T}+\frac{\partial(\boldsymbol{v} \cdot \boldsymbol{\tau})}{\partial s} \boldsymbol{\tau}^{T}+(\boldsymbol{v} \cdot \boldsymbol{\tau})\left(\frac{\partial \boldsymbol{\tau}}{\partial s}\right)^{T}\right) \\
& +\boldsymbol{n}\left(\frac{\partial(\boldsymbol{v} \cdot \boldsymbol{n})}{\partial \boldsymbol{n}} \boldsymbol{n}^{T}+(\boldsymbol{v} \cdot \boldsymbol{n})\left(\frac{\partial \boldsymbol{n}}{\partial \boldsymbol{n}}\right)^{T}+\frac{\partial(\boldsymbol{v} \cdot \boldsymbol{\tau})}{\partial \boldsymbol{n}} \boldsymbol{\tau}^{T}+(\boldsymbol{v} \cdot \boldsymbol{\tau})\left(\frac{\partial \boldsymbol{\tau}}{\partial \boldsymbol{n}}\right)^{T}\right) .
\end{aligned}
$$

Note that $\{\boldsymbol{n}, \boldsymbol{\tau}\}$ is an ortho-normal basis, $\frac{\partial \boldsymbol{n}}{\partial \boldsymbol{n}} \cdot \boldsymbol{n}=0$, and $\frac{\partial \boldsymbol{n}}{\partial s} \cdot \boldsymbol{n}=0$. Hence

$$
\begin{aligned}
(\nabla \boldsymbol{v})^{T} \boldsymbol{n} & =\boldsymbol{\tau}\left(\frac{\partial(\boldsymbol{v} \cdot \boldsymbol{n})}{\partial s}+(\boldsymbol{v} \cdot \boldsymbol{\tau})\left(\frac{\partial \boldsymbol{\tau}}{\partial s}\right)^{T} \boldsymbol{n}\right)+\boldsymbol{n}\left(\frac{\partial(\boldsymbol{v} \cdot \boldsymbol{n})}{\partial \boldsymbol{n}}+(\boldsymbol{v} \cdot \boldsymbol{\tau})\left(\frac{\partial \boldsymbol{\tau}}{\partial \boldsymbol{n}}\right)^{T} \boldsymbol{n}\right) \\
\boldsymbol{\tau} \cdot(\nabla \boldsymbol{v})^{T} \boldsymbol{n} & =\frac{\partial(\boldsymbol{v} \cdot \boldsymbol{n})}{\partial s}+(\boldsymbol{v} \cdot \boldsymbol{\tau})\left(\frac{\partial \boldsymbol{\tau}}{\partial s}\right)^{T} \boldsymbol{n} .
\end{aligned}
$$

From (2.5) and (2.6), one deduces that

$$
\begin{aligned}
2(\boldsymbol{D}(\boldsymbol{v}) \boldsymbol{n})_{\boldsymbol{\tau}} & =(\boldsymbol{\tau} \cdot(\nabla \boldsymbol{v}) \boldsymbol{n}) \boldsymbol{\tau}+\left(\boldsymbol{\tau} \cdot(\nabla \boldsymbol{v})^{T} \boldsymbol{n}\right) \boldsymbol{\tau} \\
& =\left((\boldsymbol{v} \cdot \boldsymbol{n})\left(\frac{\partial \boldsymbol{n}}{\partial \boldsymbol{n}}\right) \cdot \boldsymbol{\tau}+\frac{\partial(\boldsymbol{v} \cdot \boldsymbol{\tau})}{\partial \boldsymbol{n}}\right) \boldsymbol{\tau}+\left(\frac{\partial(\boldsymbol{v} \cdot \boldsymbol{n})}{\partial s}+(\boldsymbol{v} \cdot \boldsymbol{\tau})\left(\frac{\partial \boldsymbol{\tau}}{\partial s}\right)^{T} \boldsymbol{n}\right) \boldsymbol{\tau}
\end{aligned}
$$

Now, replacing $\boldsymbol{v}$ by $\boldsymbol{u}$ in (2.7), and using (1.5), one obtains

$$
2 \nu(\boldsymbol{D}(\boldsymbol{u}) \boldsymbol{n})_{\boldsymbol{\tau}}=\nu \frac{\partial(\boldsymbol{u} \cdot \boldsymbol{\tau})}{\partial \boldsymbol{n}} \boldsymbol{\tau}+\nu \kappa \boldsymbol{u}_{\boldsymbol{\tau}}
$$

with $\boldsymbol{u}_{\boldsymbol{\tau}}=(\boldsymbol{u} \cdot \boldsymbol{\tau}) \boldsymbol{\tau}$ and $\kappa$ being the principal curvature introduced in (2.3).

Apart from the smoothness on $S$, we make two additional assumptions regarding the shape of $\Omega$; 
(a) There are constants $\kappa_{0}, \kappa_{1}$ such that

$$
0<\kappa_{1} \leq \kappa(x) \leq \kappa_{0} \text { for all } x \in S .
$$

(b) $\Gamma$ has a positive measure, that is $|\Gamma|>0$.

Next, the vector-valued Laplace operator of a vector field $\boldsymbol{u}=\left(u_{1}, u_{2}\right)$ is given as follows

$$
\triangle \boldsymbol{u}+\nabla(\operatorname{div} \boldsymbol{u})=2 \operatorname{div} \boldsymbol{D}(\boldsymbol{u}) .
$$

\subsection{Stokes system}

In this subsection, we use the decompositions (2.8), and (2.10) to define a weak solution $\boldsymbol{u}$ to the problem $(1.1), \ldots,(1.7)$.

First from (2.10) and (1.2), (1.1) becomes

$$
-\nu \triangle \boldsymbol{u}+\nabla p \quad=\boldsymbol{f} \quad \text { in } \Omega .
$$

Let $\boldsymbol{v} \in \boldsymbol{V}_{\text {div }}$, and $\boldsymbol{u} \in \boldsymbol{V}_{\text {div }}$ solution of (1.1)-(1.7). We multiply (2.11) by $\boldsymbol{v}-\boldsymbol{u}$, integrate over $\Omega$, use Green's formula, (2.5), (2.8) to obtain

$$
\begin{aligned}
\nu \int_{\Omega} \nabla \boldsymbol{u} \cdot \nabla(\boldsymbol{v}-\boldsymbol{u}) & =\int_{\Omega} \boldsymbol{f} \cdot(\boldsymbol{v}-\boldsymbol{u})+\nu \int_{S}(\nabla \boldsymbol{u} \boldsymbol{n})_{\boldsymbol{\tau}} \cdot\left(\boldsymbol{v}_{\boldsymbol{\tau}}-\boldsymbol{u}_{\boldsymbol{\tau}}\right) \\
& =\int_{\Omega} \boldsymbol{f} \cdot(\boldsymbol{v}-\boldsymbol{u})+\nu \int_{S} \boldsymbol{\tau}(\boldsymbol{\tau} \cdot \nabla \boldsymbol{u} \boldsymbol{n}) \cdot\left(\boldsymbol{v}_{\boldsymbol{\tau}}-\boldsymbol{u}_{\boldsymbol{\tau}}\right) \\
& =\int_{\Omega} \boldsymbol{f} \cdot(\boldsymbol{v}-\boldsymbol{u})+\nu \int_{S} \boldsymbol{\tau}\left(\frac{\partial \boldsymbol{u} \cdot \boldsymbol{\tau}}{\partial \boldsymbol{n}}\right) \cdot\left(\boldsymbol{v}_{\boldsymbol{\tau}}-\boldsymbol{u}_{\boldsymbol{\tau}}\right) \\
& =\int_{\Omega} \boldsymbol{f} \cdot(\boldsymbol{v}-\boldsymbol{u})+2 \nu \int_{S}(\boldsymbol{D}(\boldsymbol{u}) \boldsymbol{n})_{\boldsymbol{\tau}} \cdot\left(\boldsymbol{v}_{\boldsymbol{\tau}}-\boldsymbol{u}_{\boldsymbol{\tau}}\right)-\nu \int_{S} \kappa \boldsymbol{u}_{\boldsymbol{\tau}} \cdot\left(\boldsymbol{v}_{\boldsymbol{\tau}}-\boldsymbol{u}_{\boldsymbol{\tau}}\right)
\end{aligned}
$$

which is re-written (see (1.6)) as: for all $\boldsymbol{v} \in \boldsymbol{V}_{\mathrm{div}}$,

$$
\begin{aligned}
\nu \int_{\Omega} \nabla \boldsymbol{u} \cdot \nabla(\boldsymbol{v}-\boldsymbol{u})+\nu \int_{S} \kappa \boldsymbol{u}_{\boldsymbol{\tau}} \cdot\left(\boldsymbol{v}_{\boldsymbol{\tau}}-\boldsymbol{u}_{\boldsymbol{\tau}}\right)= & \int_{\Omega} \boldsymbol{f} \cdot(\boldsymbol{v}-\boldsymbol{u}) \\
& +\int_{S}(\boldsymbol{T} \boldsymbol{n})_{\boldsymbol{\tau}} \cdot\left(\boldsymbol{v}_{\boldsymbol{\tau}}-\boldsymbol{u}_{\boldsymbol{\tau}}\right) .
\end{aligned}
$$

We recall that (1.8) is by definition equivalent to

$$
g\left|\boldsymbol{u}_{\boldsymbol{\tau}}\right|-g\left|\boldsymbol{v}_{\boldsymbol{\tau}}\right| \leq(\boldsymbol{T n})_{\boldsymbol{\tau}} \cdot\left(\boldsymbol{v}_{\boldsymbol{\tau}}-\boldsymbol{u}_{\boldsymbol{\tau}}\right) \text { on } S .
$$

At this juncture, we define the following functionals

$$
\begin{array}{rcrl}
a: & \boldsymbol{H}^{1}(\Omega) \times \boldsymbol{H}^{1}(\Omega) & \longrightarrow \mathbb{R} \\
& (\boldsymbol{v}, \boldsymbol{u}) & \longrightarrow a(\boldsymbol{v}, \boldsymbol{u})=\nu(\nabla \boldsymbol{v}, \nabla \boldsymbol{u})+\nu\left(\kappa \boldsymbol{u}_{\boldsymbol{\tau}}, \boldsymbol{v}_{\boldsymbol{\tau}}\right)_{S} \\
b: \quad \boldsymbol{H}^{1}(\Omega) \times M & \longrightarrow \mathbb{R} \\
& (\boldsymbol{v}, q) & \longrightarrow b(\boldsymbol{v}, q)=-(\operatorname{div} \boldsymbol{v}, q), \\
j: \quad \boldsymbol{H}^{1}(\Omega) & \longrightarrow \mathbb{R} \\
& \boldsymbol{v} & \longrightarrow j(\boldsymbol{v})=\left(g,\left|\boldsymbol{v}_{\boldsymbol{\tau}}\right|\right)_{S}, \\
\ell: \quad \boldsymbol{H}^{1}(\Omega) & \longrightarrow \mathbb{R} \\
& \boldsymbol{v} & \longrightarrow \ell(\boldsymbol{v})=(\boldsymbol{f}, \boldsymbol{v}) .
\end{array}
$$


Thus (2.12) and (2.13) leads to

$$
\left\{\begin{array}{l}
\text { Find } \boldsymbol{u} \in \boldsymbol{V}_{\mathrm{div}} \text { such that for all } \boldsymbol{v} \in \boldsymbol{V}_{\mathrm{div}} \\
a(\boldsymbol{u}, \boldsymbol{v}-\boldsymbol{u})+j(\boldsymbol{v})-j(\boldsymbol{u}) \geq \ell(\boldsymbol{v}-\boldsymbol{u}) .
\end{array}\right.
$$

One can also observe that $\boldsymbol{u}$ is the solution of the optimization problem:

$$
\left\{\begin{array}{l}
\text { Find } \boldsymbol{u} \in \boldsymbol{V}_{\mathrm{div}} \text { that satisfies, } \\
J(\boldsymbol{u}) \leq J(\boldsymbol{v}) \quad \text { for all } \quad \boldsymbol{v} \in \boldsymbol{V}_{\mathrm{div}}, \\
\text { with } J(\boldsymbol{v})=\frac{1}{2} a(\boldsymbol{v}, \boldsymbol{v})+j(\boldsymbol{v})-\ell(\boldsymbol{v}) .
\end{array}\right.
$$

Another equivalent model is the one involving the velocity and pressure, and reads

$$
\left\{\begin{array}{l}
\text { Problem }(\mathbf{P}) \\
\text { Find }(\boldsymbol{u}, p) \in \boldsymbol{V} \times M, \text { such that for all }(\boldsymbol{v}, q) \in \boldsymbol{V} \times M \\
a(\boldsymbol{u}, \boldsymbol{v}-\boldsymbol{u})+b(\boldsymbol{v}-\boldsymbol{u}, p)+j(\boldsymbol{v})-j(\boldsymbol{u}) \geq \ell(\boldsymbol{v}-\boldsymbol{u}) \\
b(\boldsymbol{u}, q)=0
\end{array}\right.
$$

Since the pressure $p$ is the Lagrange multiplier associated with the linear constraint $(2.17)_{2}$, it follows that the system (2.17) is a mixed problem. At this stage let us point out that from a numerical point of view, the solution of (2.15), or (2.16) is hard to obtain because of the difficulty to define an internal approximation of $\boldsymbol{V}_{\text {div }}$ (see [27]). Hence the mixed formulation (2.17) is introduced in order to relax the divergence free constraint in the space $\boldsymbol{V}_{\text {div }}$. In $[16,17]$, a three fields formulation is proposed, taking the tangential part of the traction force $(\boldsymbol{T n})_{\boldsymbol{\tau}}$ as unknown and exploiting the convex duality relation (1.8). Existence and uniqueness of solution of problem (2.17) has been investigated by H. FuJITA in [2, 3, 4], (see also [23, 24]). It basically entails showing that:

(a) the bilinear and linear forms $a(\cdot, \cdot), b(\cdot, \cdot)$ and $\ell$ are continuous on $\boldsymbol{V}$;

(b) $\quad j$ is convex and lower semi-continuous on $\boldsymbol{V}$;

(c) there exists a constant $\alpha>0$ such that

$$
a(\boldsymbol{v}, \boldsymbol{v}) \geq \alpha\|\boldsymbol{v}\|_{1}^{2} \text { for all } \boldsymbol{v} \in \boldsymbol{V}_{\mathrm{div}},
$$

with $\boldsymbol{V}_{\mathrm{div}}=\{\boldsymbol{w} \in \boldsymbol{V}: b(\boldsymbol{w}, q)=0$ for all $q \in M\}=\{\boldsymbol{v} \in \boldsymbol{V}: \operatorname{div} \boldsymbol{v}=0\}$

(d) there exists a constant $\beta>0$ such that

$$
\beta\|q\| \leq \sup _{\boldsymbol{v} \in \boldsymbol{V}} \frac{b(\boldsymbol{v}, q)}{\|\boldsymbol{v}\|_{1}} \text { for all } \boldsymbol{v} \in \boldsymbol{V}
$$

We then state that

Proposition 2.1 If $\boldsymbol{f}$ is an element of $\boldsymbol{L}^{2}(\Omega)$, and $g \in L^{\infty}(S)$, then the variational problem (2.17) has a unique solution $(\boldsymbol{u}, p) \in V \times M$, and the following estimates hold

$$
\|\boldsymbol{u}\|_{1} \leq \frac{\boldsymbol{c}}{\nu}\|\boldsymbol{f}\| \quad, \quad\|p\| \leq \boldsymbol{c}\|\boldsymbol{f}\|+\boldsymbol{c} \frac{\kappa_{0}}{\nu}\|\boldsymbol{f}\| .
$$


Moreover if $S$ is of class $\mathcal{C}^{3}$ and $\Gamma$ of class $\mathcal{C}^{2}$, with $g \in H^{1}(S) \cap L^{\infty}(S)$, then Saito in [5] has shown that $(\boldsymbol{u}, p) \in \boldsymbol{H}^{2}(\Omega) \times H^{1}(\Omega)$, and enjoys the a priori estimate

$$
\|\boldsymbol{u}\|_{2}+\|p\|_{1} \leq \boldsymbol{c}(\Omega, \nu)\left(\|\boldsymbol{f}\|+\|g\|_{1, S}\right) .
$$

The solution $(\boldsymbol{u}, p)$ of (2.17) is also determined by the following problem [19]: there exists a unique $\boldsymbol{\alpha} \in \Lambda$ such that

$$
\left\{\begin{array}{l}
a(\boldsymbol{u}, \boldsymbol{v})+b(\boldsymbol{v}, p)+\left(g \boldsymbol{\alpha}, \boldsymbol{v}_{\boldsymbol{\tau}}\right)_{S}=\ell(\boldsymbol{v}) \text { for all } \boldsymbol{v} \in \boldsymbol{V} \\
b(\boldsymbol{u}, q)=0 \text { for all } q \in L^{2}(\Omega) \\
\boldsymbol{\alpha} \cdot \boldsymbol{u}_{\boldsymbol{\tau}}=\left|\boldsymbol{u}_{\boldsymbol{\tau}}\right| \text { a.e on } S
\end{array}\right.
$$

with

$$
\Lambda=\left\{\boldsymbol{\alpha} \in \boldsymbol{L}^{2}(S) ;|\boldsymbol{\alpha}| \leq 1 \text { a.e. on } S\right\}
$$

The pressure $p$ is constructed as in [6], while the a priori inequality (2.20) is obtained by using (2.18) and (2.19) (see [8] for similar results).

\section{$2.3 \quad$ Navier-Stokes system}

To present the weak formulation associated with $(1.2),(1.3),(1.5),(1.7)$ and $(1.10)$, we first see from (2.10) and (1.2) that (1.10) is equivalent to

$$
-\nu \triangle \boldsymbol{u}+(\boldsymbol{u} \cdot \nabla) \boldsymbol{u}+\nabla p=\boldsymbol{f} \quad \text { in } \quad \Omega
$$

Next, we introduce the trilinear form $d(\cdot, \cdot, \cdot)$ given by

$$
d: \quad \boldsymbol{H}^{1}(\Omega) \times \boldsymbol{H}^{1}(\Omega) \times \boldsymbol{H}^{1}(\Omega) \longrightarrow \mathbb{R}, d(\boldsymbol{u}, \boldsymbol{v}, \boldsymbol{w})=\int_{\Omega}(\boldsymbol{u} \cdot \nabla) \boldsymbol{v} \cdot \boldsymbol{w} d x
$$

The trilinear form $d$ is continuous in the sense that there exists a positive constant $c$ depending on $\Omega$ such that

$$
\text { for all }(\boldsymbol{u}, \boldsymbol{v}, \boldsymbol{w}) \in \boldsymbol{V}^{3}, d(\boldsymbol{u}, \boldsymbol{v}, \boldsymbol{w}) \leq \boldsymbol{c}\|\nabla \boldsymbol{u}\|\|\nabla \boldsymbol{v}\|\|\nabla \boldsymbol{w}\|,
$$

moreover the following properties hold [34]:

$$
\begin{aligned}
d(\boldsymbol{u}, \boldsymbol{v}, \boldsymbol{w}) & =-d(\boldsymbol{u}, \boldsymbol{w}, \boldsymbol{v}) \quad \text { for all }(\boldsymbol{u}, \boldsymbol{v}, \boldsymbol{w}) \in \boldsymbol{V}_{\mathrm{div}} \times \boldsymbol{V} \times \boldsymbol{V} \\
d(\boldsymbol{u}, \boldsymbol{v}, \boldsymbol{v}) & =0 \text { for all }(\boldsymbol{u}, \boldsymbol{v}) \in \boldsymbol{V}_{\mathrm{div}} \times \boldsymbol{V}
\end{aligned}
$$

The mixed variational inequality associated to (1.2), (1.3), (1.5), (1.7) and (2.23) can be stated as follows:

$$
\left\{\begin{array}{l}
\operatorname{Problem}(\mathbf{F}) \\
\text { Find }(\boldsymbol{u}, p) \in \boldsymbol{V} \times M, \text { that such that, } \\
a(\boldsymbol{u}, \boldsymbol{v}-\boldsymbol{u})+d(\boldsymbol{u}, \boldsymbol{u}, \boldsymbol{v}-\boldsymbol{u})+b(\boldsymbol{v}-\boldsymbol{u}, p)+j(\boldsymbol{v})-j(\boldsymbol{u}) \geq \ell(\boldsymbol{v}-\boldsymbol{u}) \\
b(\boldsymbol{u}, q)=0 \\
\text { for all }(\boldsymbol{v}, q) \in \boldsymbol{V} \times M
\end{array}\right.
$$

The solvability of problem (F) is obtained by combining; Galerkin's approximation, monotone operator theory, compactness arguments. In fact one claims that 
Theorem 2.1 Let $(\boldsymbol{f}, g) \in \boldsymbol{L}^{2}(\Omega) \times L^{\infty}(S)$, with $\nu$ taken such that

$$
\nu-\frac{\boldsymbol{c}}{\nu}\|\boldsymbol{f}\| \geq 0
$$

where $\boldsymbol{c}$ is a generic positive constant depending only of $\Omega$. Then problem (2.27) is uniquely solvable and the following hold

$$
\begin{aligned}
\|\nabla \boldsymbol{u}\| & \leq \frac{\boldsymbol{c}}{\nu}\|\boldsymbol{f}\| \\
\|p\| & \leq \boldsymbol{c}\|\boldsymbol{f}\|+\nu \boldsymbol{c}\|\boldsymbol{f}\|+\boldsymbol{c} \nu \kappa_{0}\|\boldsymbol{f}\|+\frac{\boldsymbol{c}}{\nu^{2}}\|\boldsymbol{f}\|^{2}
\end{aligned}
$$

Proof. The solution $(\boldsymbol{u}, p)$ solution of problem (F) is constructed in several steps.

step 1: Regularization

Since the functional $j$ is non differentiable at zero, we approximate it by $j_{\varepsilon}$ with

$$
j_{\varepsilon}(\boldsymbol{v})=\left(g, \sqrt{\left|\boldsymbol{v}_{\boldsymbol{\tau}}\right|^{2}+\varepsilon^{2}}\right)_{S} .
$$

The functional $J_{\varepsilon}$ is convex, lower semi-continuous and Gateaux-derivative with

$$
\begin{aligned}
\left\langle j_{\varepsilon}^{\prime}(\boldsymbol{u}), \boldsymbol{v}\right\rangle & =\int_{S} g \frac{\boldsymbol{u}_{\boldsymbol{\tau}} \cdot \boldsymbol{v}_{\boldsymbol{\tau}}}{\sqrt{\left|\boldsymbol{u}_{\boldsymbol{\tau}}\right|^{2}+\varepsilon^{2}}}, \\
j_{\varepsilon}^{(2)}(\boldsymbol{u})(\boldsymbol{v}, \boldsymbol{w}) & =\int_{S} g \frac{\left(\boldsymbol{v}_{\boldsymbol{\tau}} \cdot \boldsymbol{w}_{\boldsymbol{\tau}}\right)\left(\left|\boldsymbol{u}_{\boldsymbol{\tau}}\right|^{2}+\varepsilon^{2}\right)-\left(\boldsymbol{u}_{\boldsymbol{\tau}} \cdot \boldsymbol{w}_{\boldsymbol{\tau}}\right)\left(\boldsymbol{u}_{\boldsymbol{\tau}} \cdot \boldsymbol{v}_{\boldsymbol{\tau}}\right)}{\left(\left|\boldsymbol{u}_{\boldsymbol{\tau}}\right|^{2}+\varepsilon^{2}\right)^{3 / 2}} .
\end{aligned}
$$

Observe that $j^{(2)}$ is symmetric

$$
\text { for all } \boldsymbol{v}, \boldsymbol{w} \in \boldsymbol{H}^{1}(\Omega), \quad j_{\varepsilon}^{(2)}(\boldsymbol{u})(\boldsymbol{v}, \boldsymbol{w})=j_{\varepsilon}^{(2)}(\boldsymbol{u})(\boldsymbol{w}, \boldsymbol{v}),
$$

and positive definite: for all $\boldsymbol{v} \in \boldsymbol{H}^{1}(\Omega)$

$$
\begin{aligned}
j_{\varepsilon}^{(2)}(\boldsymbol{u})(\boldsymbol{v}, \boldsymbol{v}) & =\int_{S} g \frac{\left|\boldsymbol{v}_{\boldsymbol{\tau}}\right|^{2}\left(\left|\boldsymbol{u}_{\boldsymbol{\tau}}\right|^{2}+\varepsilon^{2}\right)-\left(\boldsymbol{u}_{\boldsymbol{\tau}} \cdot \boldsymbol{v}_{\boldsymbol{\tau}}\right)\left(\boldsymbol{u}_{\boldsymbol{\tau}} \cdot \boldsymbol{v}_{\boldsymbol{\tau}}\right)}{\left(\left|\boldsymbol{u}_{\boldsymbol{\tau}}\right|^{2}+\varepsilon^{2}\right)^{3 / 2}} \\
& \geq \int_{S} g \frac{\left|\boldsymbol{v}_{\boldsymbol{\tau}}\right|^{2}\left(\left|\boldsymbol{u}_{\boldsymbol{\tau}}\right|^{2}+\varepsilon^{2}\right)-\left|\boldsymbol{u}_{\boldsymbol{\tau}}\right|^{2}\left|\boldsymbol{v}_{\boldsymbol{\tau}}\right|^{2}}{\left(\left|\boldsymbol{u}_{\boldsymbol{\tau}}\right|^{2}+\varepsilon^{2}\right)^{3 / 2}} \\
& =\int_{S} g \frac{\varepsilon^{2}\left|\boldsymbol{v}_{\boldsymbol{\tau}}\right|^{2}}{\left(\left|\boldsymbol{u}_{\boldsymbol{\tau}}\right|^{2}+\varepsilon^{2}\right)^{3 / 2}}>0
\end{aligned}
$$

The regularized problem reads:

$$
\left\{\begin{array}{l}
\text { Find } \boldsymbol{u}_{\varepsilon} \in \boldsymbol{V}_{\mathrm{div}}, \text { that such that, } \\
a\left(\boldsymbol{u}_{\varepsilon}, \boldsymbol{v}-\boldsymbol{u}_{\varepsilon}\right)+d\left(\boldsymbol{u}_{\varepsilon}, \boldsymbol{u}_{\varepsilon}, \boldsymbol{v}-\boldsymbol{u}_{\varepsilon}\right)+j_{\varepsilon}(\boldsymbol{v})-j_{\varepsilon}\left(\boldsymbol{u}_{\varepsilon}\right) \geq \ell\left(\boldsymbol{v}-\boldsymbol{u}_{\varepsilon}\right) \\
\text { for all } \boldsymbol{v} \in \boldsymbol{V}_{\operatorname{div}}
\end{array}\right.
$$

Using some classical arguments in [9] (see page 157-158), it turn out that the problem (2.31) is equivalent to

$$
\left\{\begin{array}{l}
\text { Find } \boldsymbol{u}_{\varepsilon} \in \boldsymbol{V}_{\mathrm{div}}, \text { that such that, } \\
a\left(\boldsymbol{u}_{\varepsilon}, \boldsymbol{v}\right)+d\left(\boldsymbol{u}_{\varepsilon}, \boldsymbol{u}_{\varepsilon}, \boldsymbol{v}\right)+\left\langle j_{\varepsilon}^{\prime}\left(\boldsymbol{u}_{\varepsilon}\right), \boldsymbol{v}\right\rangle=\ell(\boldsymbol{v}), \\
\text { for all } \boldsymbol{v} \in \boldsymbol{V}_{\mathrm{div}}
\end{array}\right.
$$


step 2. Galerkin approximation.

We recall since $\boldsymbol{V}_{\text {div }}$ is a separable Hilbert space, one can find $\left\{\boldsymbol{\phi}_{i}\right\}_{i=1}^{\infty}$ an orthonormal basis of $\boldsymbol{V}_{\text {div }}$, with

$$
\boldsymbol{W}_{n}=\left\{\phi_{1}, \phi_{2}, \ldots, \phi_{n}\right\}, \quad \overline{\boldsymbol{W}}_{n}=\boldsymbol{V}_{\mathrm{div}} .
$$

Then one considers the Galerkin problem;

$$
\left\{\begin{array}{l}
\text { Find } \boldsymbol{u}_{\varepsilon}^{n} \in \boldsymbol{W}_{n}, \text { that such that, } \\
a\left(\boldsymbol{u}_{\varepsilon}^{n}, \boldsymbol{v}\right)+d\left(\boldsymbol{u}_{\varepsilon}^{n}, \boldsymbol{u}_{\varepsilon}^{n}, \boldsymbol{v}\right)+\left\langle j_{\varepsilon}^{\prime}\left(\boldsymbol{u}_{\varepsilon}^{n}\right), \boldsymbol{v}\right\rangle=\ell(\boldsymbol{v}) \\
\text { for all } \boldsymbol{v} \in \boldsymbol{W}_{n} .
\end{array}\right.
$$

To study (2.33) it is convenient to introduce the mapping $\boldsymbol{u} \mapsto \Phi(\boldsymbol{u})$ defined by

$$
(\Phi(\boldsymbol{u}) \mid \boldsymbol{v})=a(\boldsymbol{u}, \boldsymbol{v})+d(\boldsymbol{u}, \boldsymbol{u}, \boldsymbol{v})+\left\langle j_{\varepsilon}^{\prime}(\boldsymbol{u}), \boldsymbol{v}\right\rangle .
$$

Then $\Phi$ maps $\boldsymbol{W}_{n}$ into $\boldsymbol{W}_{n}$ with the $\boldsymbol{H}^{1}(\Omega)$-norm, and is bounded on all bounded subsets of $\boldsymbol{H}^{1}(\Omega)$; Indeed for $\boldsymbol{u}, \boldsymbol{v} \in \boldsymbol{W}_{n}$,

$$
\begin{aligned}
(\Phi(\boldsymbol{u}) \mid \boldsymbol{v}) & \leq \nu\|\nabla \boldsymbol{u}\|\|\nabla \boldsymbol{v}\|+\nu \kappa_{0}\left\|\boldsymbol{u}_{\boldsymbol{\tau}}\right\|_{S}\left\|\boldsymbol{v}_{\boldsymbol{\tau}}\right\|_{S}+\boldsymbol{c}\|\boldsymbol{u}\|\|\nabla \boldsymbol{u}\|\|\nabla \boldsymbol{v}\|+\frac{1}{\varepsilon} \int_{S} g \boldsymbol{u}_{\boldsymbol{\tau}} \cdot \boldsymbol{v}_{\boldsymbol{\tau}} \\
& \leq \nu\|\nabla \boldsymbol{u}\|\|\nabla \boldsymbol{v}\|+\nu \boldsymbol{c} \kappa_{0}\|\nabla \boldsymbol{u}\|\|\nabla \boldsymbol{v}\|+\boldsymbol{c}\|\nabla \boldsymbol{u}\|^{2}\|\nabla \boldsymbol{v}\|+\frac{\boldsymbol{c}}{\varepsilon}\|g\|_{L^{\infty}(S)}\|\nabla \boldsymbol{u}\|\|\nabla \boldsymbol{v}\|
\end{aligned}
$$

from which we deduce that

$$
\|\Phi(\boldsymbol{u})\|_{1} \leq \nu\|\boldsymbol{u}\|_{1}+\nu \boldsymbol{c} \kappa_{0}\|\boldsymbol{u}\|_{1}+\boldsymbol{c}\|\boldsymbol{u}\|_{1}^{2}+\frac{\boldsymbol{c}}{\varepsilon}\|g\|_{L^{\infty}(S)}\|\boldsymbol{u}\|_{1}
$$

The discrete system (2.33) is a nonlinear problem in which one of the nonlinearity is of monotone type. Hence for its solvability, one should prove that: $\Phi$ is monotone, coercive, and hemicontinuous.

Observe that if $\boldsymbol{u}_{\varepsilon}^{n}$ is the solution of (2.33), then for $\boldsymbol{v}=\boldsymbol{u}_{\varepsilon}^{n}$ one has

$$
a\left(\boldsymbol{u}_{\varepsilon}^{n}, \boldsymbol{u}_{\varepsilon}^{n}\right)+d\left(\boldsymbol{u}_{\varepsilon}^{n}, \boldsymbol{u}_{\varepsilon}^{n}, \boldsymbol{u}_{\varepsilon}^{n}\right)+\left\langle j_{\varepsilon}^{\prime}\left(\boldsymbol{u}_{\varepsilon}^{n}\right), \boldsymbol{u}_{\varepsilon}^{n}\right\rangle=\ell\left(\boldsymbol{u}_{\varepsilon}^{n}\right)
$$

which from the fact that $j_{\varepsilon}^{\prime}$ is monotone, and $d\left(\boldsymbol{u}_{\varepsilon}^{n}, \boldsymbol{u}_{\varepsilon}^{n}, \boldsymbol{u}_{\varepsilon}^{n}\right)=0$ leads to

$$
\nu\left\|\nabla \boldsymbol{u}_{\varepsilon}^{n}\right\|^{2}+\nu \kappa_{1}\left\|\boldsymbol{u}_{\varepsilon, \boldsymbol{\tau}}^{n}\right\|_{S}^{2} \leq\|\boldsymbol{f}\|\left\|\boldsymbol{u}_{\varepsilon}^{n}\right\| \leq \boldsymbol{c} \frac{\|\boldsymbol{f}\|^{2}}{2 \nu}+\frac{\nu}{2}\left\|\nabla \boldsymbol{u}_{\varepsilon}^{n}\right\|^{2} .
$$

Thus

$$
\left\|\nabla \boldsymbol{u}_{\varepsilon}^{n}\right\| \leq \frac{\boldsymbol{c}}{\nu}\|\boldsymbol{f}\|
$$

- $\Phi$ is monotone. Indeed for $\boldsymbol{u}_{1}, \boldsymbol{u}_{2} \in \boldsymbol{W}_{n}$ such that (2.36) holds. Then

$$
\begin{aligned}
& \left(\Phi\left(\boldsymbol{u}_{1}\right)-\Phi\left(\boldsymbol{u}_{2}\right) \mid \boldsymbol{u}_{1}-\boldsymbol{u}_{2}\right) \\
= & a\left(\boldsymbol{u}_{1}-\boldsymbol{u}_{2}, \boldsymbol{u}_{1}-\boldsymbol{u}_{2}\right)+d\left(\boldsymbol{u}_{1}, \boldsymbol{u}_{1}, \boldsymbol{u}_{1}-\boldsymbol{u}_{2}\right)-d\left(\boldsymbol{u}_{2}, \boldsymbol{u}_{2}, \boldsymbol{u}_{1}-\boldsymbol{u}_{2}\right) \\
& +\left\langle j_{\varepsilon}^{\prime}\left(\boldsymbol{u}_{1}\right), \boldsymbol{u}_{1}-\boldsymbol{u}_{2}\right\rangle-\left\langle j_{\varepsilon}^{\prime}\left(\boldsymbol{u}_{2}\right), \boldsymbol{u}_{1}-\boldsymbol{u}_{2}\right\rangle \\
= & a\left(\boldsymbol{u}_{1}-\boldsymbol{u}_{2}, \boldsymbol{u}_{1}-\boldsymbol{u}_{2}\right)+d\left(\boldsymbol{u}_{1}-\boldsymbol{u}_{2}, \boldsymbol{u}_{2}, \boldsymbol{u}_{1}-\boldsymbol{u}_{2}\right)+\left\langle j_{\varepsilon}^{\prime}\left(\boldsymbol{u}_{1}\right)-j_{\varepsilon}^{\prime}\left(\boldsymbol{u}_{2}\right), \boldsymbol{u}_{1}-\boldsymbol{u}_{2}\right\rangle \\
= & a\left(\boldsymbol{u}_{1}-\boldsymbol{u}_{2}, \boldsymbol{u}_{1}-\boldsymbol{u}_{2}\right)+d\left(\boldsymbol{u}_{1}-\boldsymbol{u}_{2}, \boldsymbol{u}_{2}, \boldsymbol{u}_{1}-\boldsymbol{u}_{2}\right)+\int_{0}^{1} j_{\varepsilon}^{(2)}\left(\boldsymbol{u}_{2}+\theta\left(\boldsymbol{u}_{1}-\boldsymbol{u}_{2}\right)\right) \cdot\left(\boldsymbol{u}_{1}-\boldsymbol{u}_{2}, \boldsymbol{u}_{1}-\boldsymbol{u}_{2}\right) d \theta \\
\geq & \nu\left\|\nabla\left(\boldsymbol{u}_{1}-\boldsymbol{u}_{2}\right)\right\|^{2}-\boldsymbol{c}\left\|\nabla\left(\boldsymbol{u}_{1}-\boldsymbol{u}_{2}\right)\right\|^{2}\left\|\nabla \boldsymbol{u}_{2}\right\|+\int_{0}^{1} j_{\varepsilon}^{(2)}\left(\boldsymbol{u}_{2}+\theta\left(\boldsymbol{u}_{1}-\boldsymbol{u}_{2}\right)\right) \cdot\left(\boldsymbol{u}_{1}-\boldsymbol{u}_{2}, \boldsymbol{u}_{1}-\boldsymbol{u}_{2}\right) d \theta \\
\geq & \left(\nu-\frac{\boldsymbol{c}}{\nu}\|\boldsymbol{f}\|\right)\left\|\nabla\left(\boldsymbol{u}_{1}-\boldsymbol{u}_{2}\right)\right\|^{2}+\int_{0}^{1} \underbrace{j_{\varepsilon}^{(2)}\left(\boldsymbol{u}_{2}+\theta\left(\boldsymbol{u}_{1}-\boldsymbol{u}_{2}\right)\right) \cdot\left(\boldsymbol{u}_{1}-\boldsymbol{u}_{2}, \boldsymbol{u}_{1}-\boldsymbol{u}_{2}\right)}_{\geq 0, \text { see }(2.30)} d \theta .
\end{aligned}
$$


Thus $\Phi$ is monotone thanks to (2.28).

- $\Phi$ is coercive, that is

$$
\lim _{\|\boldsymbol{u}\|_{1} \rightarrow \infty}\left[\frac{1}{\|\boldsymbol{u}\|_{1}}(\Phi(\boldsymbol{u}) \mid \boldsymbol{u})\right]=\infty
$$

Observing that $d(\boldsymbol{u}, \boldsymbol{u}, \boldsymbol{u})=0$ and $\left\langle j_{\varepsilon}^{\prime}(\boldsymbol{u}), \boldsymbol{u}\right\rangle \geq 0$, One has

$$
\begin{aligned}
(\Phi(\boldsymbol{u}) \mid \boldsymbol{u}) & =a(\boldsymbol{u}, \boldsymbol{u})+d(\boldsymbol{u}, \boldsymbol{u}, \boldsymbol{u})+\left\langle j_{\varepsilon}^{\prime}(\boldsymbol{u}), \boldsymbol{u}\right\rangle \\
& =a(\boldsymbol{u}, \boldsymbol{u})+\left\langle j_{\varepsilon}^{\prime}(\boldsymbol{u}), \boldsymbol{u}\right\rangle \\
& \geq \nu\|\nabla \boldsymbol{u}\|^{2}+\nu \kappa_{1}\left\|\boldsymbol{u}_{\boldsymbol{\tau}}\right\|_{S}^{2}>\nu\|\nabla \boldsymbol{u}\|^{2} .
\end{aligned}
$$

Hence $\Phi$ is coercive.

- $\Phi$ is hemi-continuous, that is for $\boldsymbol{u}, \boldsymbol{v}$ in $\boldsymbol{W}_{n}$

$$
t \rightarrow(\Phi(\boldsymbol{u}+t \boldsymbol{v}) \mid \boldsymbol{v}) \text { is continuous. }
$$

Let $t_{1}, t_{2}$ in $\mathbb{R}$, then

$$
\begin{aligned}
& \left(\Phi\left(\boldsymbol{u}+t_{1} \boldsymbol{v}\right)-\Phi\left(\boldsymbol{u}+t_{2} \boldsymbol{v}\right) \mid \boldsymbol{v}\right) \\
= & \left(t_{1}-t_{2}\right) a(\boldsymbol{v}, \boldsymbol{v})+\left(t_{1}-t_{2}\right) d\left(\boldsymbol{v}, \boldsymbol{u}+t_{1} \boldsymbol{v}, \boldsymbol{v}\right)+\left\langle j_{\varepsilon}^{\prime}\left(\boldsymbol{u}+t_{1} \boldsymbol{v}\right)-j_{\varepsilon}^{\prime}\left(\boldsymbol{u}+t_{2} \boldsymbol{v}\right), \boldsymbol{v}\right\rangle \\
= & \left(t_{1}-t_{2}\right) a(\boldsymbol{v}, \boldsymbol{v})+\left(t_{1}-t_{2}\right) d\left(\boldsymbol{v}, \boldsymbol{u}+t_{1} \boldsymbol{v}, \boldsymbol{v}\right)+\left(t_{1}-t_{2}\right) \int_{0}^{1} j_{\varepsilon}^{(2)}\left(\boldsymbol{u}-t_{2} \boldsymbol{v}-\theta\left(t_{2}-t_{1}\right) \boldsymbol{v}\right)(\boldsymbol{v}, \boldsymbol{v}) d \theta \\
= & \left(t_{1}-t_{2}\right)\left[a(\boldsymbol{v}, \boldsymbol{v})+d\left(\boldsymbol{v}, \boldsymbol{u}+t_{1} \boldsymbol{v}, \boldsymbol{v}\right)+\int_{0}^{1} j_{\varepsilon}^{(2)}\left(\boldsymbol{u}-t_{2} \boldsymbol{v}-\theta\left(t_{2}-t_{1}\right) \boldsymbol{v}\right)(\boldsymbol{v}, \boldsymbol{v}) d \theta\right] .
\end{aligned}
$$

Since $\boldsymbol{v}, \boldsymbol{u}$ are fixed, it follows from (2.29) that the right hand side term in (2.38) tends to zero with $t_{1}-t_{2}$.

We conclude that (2.33) has only one solution.

step 4: a priori estimates and passage to the limit.

We recall that the inequality $(2.36)$

$$
\left\|\nabla \boldsymbol{u}_{\varepsilon}^{n}\right\| \leq \frac{\boldsymbol{c}}{\nu}\|\boldsymbol{f}\|
$$

We then deduce that $\boldsymbol{u}_{\varepsilon}^{n}$ is bounded in $\boldsymbol{H}^{1}(\Omega)$ by a constant $\boldsymbol{c}$ independent of $n$ and $\varepsilon$. Now owing to the imbedding of $H^{1}(\Omega)$ into $L^{4}(\Omega)$, there exists a subsequence, still denoted by $\left(\boldsymbol{u}_{\varepsilon}^{n}\right)_{n}$ for convenience, which converges to $\boldsymbol{u}_{\varepsilon}^{n}$ weakly in $\boldsymbol{H}^{1}(\Omega)$ and strongly in $\boldsymbol{L}^{4}(\Omega)$.

With equation (2.31) written in $\boldsymbol{W}_{n}$, passing to the limit on $n$ is obvious for linear expression, while for the nonlinear expression $\left(\boldsymbol{u}_{\varepsilon}^{n} \cdot \nabla\right) \boldsymbol{u}_{\varepsilon}^{n}$ we will use the strong convergence in $\boldsymbol{L}^{4}(\Omega)$. We also recall that since $j_{\varepsilon}$ is convex, and l.s.c

$$
\boldsymbol{u}_{\varepsilon}^{n} \rightarrow \boldsymbol{u}_{\varepsilon} \text { weakly in } \boldsymbol{V}_{\text {div }} \text {, implies that } J_{\varepsilon}\left(\boldsymbol{u}_{\varepsilon}\right) \leq \lim _{n \rightarrow \infty} \inf J_{\varepsilon}\left(\boldsymbol{u}_{\varepsilon}^{n}\right) .
$$

Thus the regularized velocity $\boldsymbol{u}_{\varepsilon}$ satisfying (2.31) is constructed. As far as the regularized pressure $p_{\varepsilon}$ is concerned, we follow $[8]$ and let

$$
\boldsymbol{v} \in \mathbb{V} \rightarrow H\left(\boldsymbol{u}_{\varepsilon}\right) \boldsymbol{v}=a\left(\boldsymbol{u}_{\varepsilon}, \boldsymbol{v}\right)+d\left(\boldsymbol{u}_{\varepsilon}, \boldsymbol{u}_{\varepsilon}, \boldsymbol{v}\right)+\left\langle j_{\varepsilon}^{\prime}\left(\boldsymbol{u}_{\varepsilon}\right), \boldsymbol{v}\right\rangle-\ell(\boldsymbol{v}) .
$$

$H\left(\boldsymbol{u}_{\varepsilon}\right)(\cdot)$ is a linear and continuous application that vanishes on $\mathbb{V}_{\text {div }}$ if $\boldsymbol{u}_{\varepsilon}$ the regularized pressure given by

$$
\left\{\begin{array}{l}
a\left(\boldsymbol{u}_{\varepsilon}, \boldsymbol{v}\right)+d\left(\boldsymbol{u}_{\varepsilon}, \boldsymbol{u}_{\varepsilon}, \boldsymbol{v}\right)+\left\langle j_{\varepsilon}^{\prime}\left(\boldsymbol{u}_{\varepsilon}\right), \boldsymbol{v}\right\rangle=\ell(\boldsymbol{v}), \\
\text { for all } \boldsymbol{v} \in \boldsymbol{V}_{\mathrm{div}}
\end{array}\right.
$$


Hence there is $p_{\varepsilon} \in M$ such that

$$
\left\{\begin{array}{l}
a\left(\boldsymbol{u}_{\varepsilon}, \boldsymbol{v}\right)+d\left(\boldsymbol{u}_{\varepsilon}, \boldsymbol{u}_{\varepsilon}, \boldsymbol{v}\right)+b\left(\boldsymbol{v}, p_{\varepsilon}\right)+\left\langle j_{\varepsilon}^{\prime}\left(\boldsymbol{u}_{\varepsilon}\right), \boldsymbol{v}\right\rangle=\ell(\boldsymbol{v}), \\
b\left(\boldsymbol{u}_{\varepsilon}, q\right)=0 \\
\text { for all }(\boldsymbol{v}, q) \in \boldsymbol{V} \times M
\end{array}\right.
$$

which is equivalent to

$$
\left\{\begin{array}{l}
\text { for all }(\boldsymbol{v}, q) \in \mathbb{V} \times M \\
a\left(\boldsymbol{u}_{\varepsilon}, \boldsymbol{v}-\boldsymbol{u}_{\varepsilon}\right)+b\left(\boldsymbol{v}-\boldsymbol{u}_{\varepsilon}, p_{\varepsilon}\right)+d\left(\boldsymbol{u}_{\varepsilon}, \boldsymbol{u}_{\varepsilon}, \boldsymbol{v}-\boldsymbol{u}_{\varepsilon}\right)+j(\boldsymbol{v})-j\left(\boldsymbol{u}_{\varepsilon}\right) \geq \ell\left(\boldsymbol{v}-\boldsymbol{u}_{\varepsilon}\right), \\
b\left(\boldsymbol{u}_{\varepsilon}, q\right)=0
\end{array}\right.
$$

In order to pass to the limit in (2.40) we need first some a priori estimates on $\boldsymbol{u}_{\varepsilon}$ and $p_{\varepsilon}$. From the estimate (2.36), we deduce that

$$
\left\|\nabla \boldsymbol{u}_{\varepsilon}\right\| \leq \frac{\boldsymbol{c}}{\nu}\|\boldsymbol{f}\| .
$$

We next derive a priori estimate for the pressure. For that purpose, we replace $\boldsymbol{v}-\boldsymbol{u}_{\varepsilon}$ in (2.41) by $\pm \boldsymbol{w} \in \boldsymbol{V}$ with $\left.\boldsymbol{w}\right|_{S}=\mathbf{0}$. One obtains

$$
b\left(\boldsymbol{w}, p_{\varepsilon}\right)=\ell(\boldsymbol{w})-a\left(\boldsymbol{u}_{\varepsilon}, \boldsymbol{w}\right)-d\left(\boldsymbol{u}_{\varepsilon}, \boldsymbol{u}_{\varepsilon}, \boldsymbol{w}\right) .
$$

The compatibility condition between $\boldsymbol{V}$ and $M$ together with (2.43) and Cauchy-Shwarz's inequality and (2.42) lead to

$$
\begin{aligned}
\beta\left\|p_{\varepsilon}\right\| & \leq \sup _{\boldsymbol{w} \in \boldsymbol{V},\left.\boldsymbol{w}\right|_{S}=0} \frac{b\left(\boldsymbol{w}, p_{\varepsilon}\right)}{\|\boldsymbol{w}\|_{1}} \\
& =\sup _{\boldsymbol{w} \in \boldsymbol{V},\left.\boldsymbol{w}\right|_{S}=0} \frac{\ell(\boldsymbol{w})-a\left(\boldsymbol{u}_{\varepsilon}, \boldsymbol{w}\right)-d\left(\boldsymbol{u}_{\varepsilon}, \boldsymbol{u}_{\varepsilon}, \boldsymbol{w}\right)}{\|\boldsymbol{w}\|_{1}} \\
& \leq\|\boldsymbol{f}\|+\nu\left\|\nabla \boldsymbol{u}_{\varepsilon}\right\|+\nu \kappa_{0}\left\|\nabla \boldsymbol{u}_{\varepsilon}\right\|+\boldsymbol{c}\left\|\nabla \boldsymbol{u}_{\varepsilon}\right\|^{2} \\
& \leq\|\boldsymbol{f}\|+\nu \boldsymbol{c}\|\boldsymbol{f}\|+\boldsymbol{c} \nu \kappa_{0}\|\boldsymbol{f}\|+\frac{\boldsymbol{c}}{\nu^{2}}\|\boldsymbol{f}\|^{2} .
\end{aligned}
$$

Since the bounds in (2.42) and (2.44) are independent of $\varepsilon$, we can repeat the analysis when passing to the limit with $n$ here, and we conclude the existence of $(\boldsymbol{u}, p) \in \mathbb{V} \times M$ such that

$$
\left\{\begin{array}{l}
\text { for all } \boldsymbol{v}, q \in \mathbb{V} \times M, \\
a(\boldsymbol{u}, \boldsymbol{v}-\boldsymbol{u})+b(\boldsymbol{v}-\boldsymbol{u}, p)+d(\boldsymbol{u}, \boldsymbol{u}, \boldsymbol{v}-\boldsymbol{u})+j(\boldsymbol{v})-j(\boldsymbol{u}) \geq \ell(\boldsymbol{v}-\boldsymbol{u}), \\
b(\boldsymbol{u}, q)=0
\end{array}\right.
$$

Moreover we have

$$
\begin{aligned}
\|\nabla \boldsymbol{u}\| & \leq \frac{\boldsymbol{c}}{\nu}\|\boldsymbol{f}\|, \\
\|p\| & \leq \boldsymbol{c}\|\boldsymbol{f}\|+\nu \boldsymbol{c}\|\boldsymbol{f}\|+\boldsymbol{c} \nu \kappa_{0}\|\boldsymbol{f}\|+\frac{\boldsymbol{c}}{\nu^{2}}\|\boldsymbol{f}\|^{2} .
\end{aligned}
$$

step 6. Uniqueness

Let $\boldsymbol{u}_{1}$ and $\boldsymbol{u}_{2}$ solutions of (2.33). Using (2.26), (2.24), the coercivity of $a(\cdot, \cdot)$, and the inequality 
(2.42) one obtains

$$
\begin{aligned}
\nu c\left\|\boldsymbol{u}_{1}-\boldsymbol{u}_{2}\right\|_{1}^{2} & \leq a\left(\boldsymbol{u}_{1}-\boldsymbol{u}_{2}, \boldsymbol{u}_{1}-\boldsymbol{u}_{2}\right) \\
& =a\left(\boldsymbol{u}_{1}, \boldsymbol{u}_{1}-\boldsymbol{u}_{2}\right)-a\left(\boldsymbol{u}_{2}, \boldsymbol{u}_{1}-\boldsymbol{u}_{2}\right) \\
& \leq d\left(\boldsymbol{u}_{1}, \boldsymbol{u}_{1}, \boldsymbol{u}_{2}-\boldsymbol{u}_{1}\right)-d\left(\boldsymbol{u}_{2}, \boldsymbol{u}_{2}, \boldsymbol{u}_{2}-\boldsymbol{u}_{1}\right) \\
& =d\left(\boldsymbol{u}_{1}, \boldsymbol{u}_{1}, \boldsymbol{u}_{2}-\boldsymbol{u}_{1}\right)-d\left(\boldsymbol{u}_{2}, \boldsymbol{u}_{1}, \boldsymbol{u}_{2}-\boldsymbol{u}_{1}\right) \\
& =d\left(\boldsymbol{u}_{1}-\boldsymbol{u}_{2}, \boldsymbol{u}_{1}, \boldsymbol{u}_{2}-\boldsymbol{u}_{1}\right) \\
& \leq \boldsymbol{c}_{0}\left\|\boldsymbol{u}_{1}-\boldsymbol{u}_{2}\right\|_{1}^{2}\left\|\boldsymbol{u}_{1}\right\|_{1} \leq \frac{\boldsymbol{c}_{0}}{\nu}\|\boldsymbol{f}\|\left\|\boldsymbol{u}_{1}-\boldsymbol{u}_{2}\right\|_{1}^{2} .
\end{aligned}
$$

Hence assuming (2.28), one sees that $\boldsymbol{u}_{1}=\boldsymbol{u}_{2}$.

So the proof is complete

The explicit dependence with respect to $\nu$ of the constant appearing in (2.42) is crucial of determining the condition for the uniqueness of solution.

Now, about the regularity of the weak solution of Problem (F) constructed in Theorem 2.1, we follow Saito [5], and claim that

Proposition 2.2 If $S$ is of class $\mathcal{C}^{3}$ and $\Gamma$ of class $\mathcal{C}^{2}$, with $g \in L^{\infty}(S) \cap H^{1}(S)$, and assuming (2.28), then $(\boldsymbol{u}, p) \in \boldsymbol{H}^{2}(\Omega) \times H^{1}(\Omega)$, and enjoys the a priori estimate

$$
\|\boldsymbol{u}\|_{2}+\|p\|_{1} \leq \boldsymbol{c}(\Omega, \nu)\left(\|\boldsymbol{f}\|+\|g\|_{1, S}\right) .
$$

The solution $(\boldsymbol{u}, p)$ of (2.27) is also determined by the following problem [19]: there exists a unique $\boldsymbol{\alpha} \in \Lambda$ such that

$$
\left\{\begin{array}{l}
a(\boldsymbol{u}, \boldsymbol{v})+d(\boldsymbol{u}, \boldsymbol{u}, \boldsymbol{v})+b(\boldsymbol{v}, p)+\left(g \boldsymbol{\alpha}, \boldsymbol{v}_{\boldsymbol{\tau}}\right)_{S}=\ell(\boldsymbol{v}) \text { for all } \boldsymbol{v} \in \boldsymbol{V} \\
b(\boldsymbol{u}, q)=0 \text { for all } q \in L^{2}(\Omega) \\
\boldsymbol{\alpha} \cdot \boldsymbol{u}_{\boldsymbol{\tau}}=\left|\boldsymbol{u}_{\boldsymbol{\tau}}\right| \text { a.e on } S
\end{array}\right.
$$

with

$$
\Lambda=\left\{\boldsymbol{\alpha} \in \boldsymbol{L}^{2}(S) ;|\boldsymbol{\alpha}| \leq 1 \text { a.e. on } S\right\}
$$

\subsection{Non conforming approximation: Some preliminaries}

In this work, we will consider both finite element approximations associated with (2.17) and (2.27), and our aim is to study their convergence.

To start with, the domain $\Omega$ is a polygon and $\bar{\Gamma} \cap \bar{S} \neq \emptyset$. It should be made clear that the regularity result about the solution $(\boldsymbol{u}, p)$ obtained by Saito [5] has not yet been shown in this situation. Hence in Proposition 3.2, Proposition 3.3 and Proposition 4.2 we assume that $(\boldsymbol{u}, p)$ belong to $\boldsymbol{H}^{2}(\Omega) \times H^{1}(\Omega)$.

Because on the assumption on $\Omega$, its closure $\bar{\Omega}$ is completely recovered by a finite number of closed triangles with disjoint interiors, that is

$$
\bar{\Omega}=\bigcup_{i=1}^{N} K_{i} .
$$

We denote by $\mathcal{T}$ the triangulation described and enforce that two elements of $\mathcal{T}$ are either disjoints, or share exactly one common vertex or edge. We denote by $h_{K}$ the diameter of $K \in \mathcal{T}$, and $\rho_{K}$ the diameter of the circle inscribed in $K$, and finally let

$$
h=\max \left\{h_{K}, \quad K \in \mathcal{T}\right\} .
$$


We also assume that the triangulation $\mathcal{T}$ is regular (also called non-degenerate) in the sense of Ciarlet [28]; that is, there exists a constant $\sigma$, independent of $h$ and $K$, such that

$$
\text { for all } K \in \mathcal{T}, \frac{h_{K}}{\rho_{K}}=\sigma_{K} \leq \sigma .
$$

For the sake of convenience, one introduces the broken Sobolev space (let $m$ be a natural number)

$$
H^{m}(\Omega ; \mathcal{T})=\left\{v \in L^{2}(\Omega) ;\left.v\right|_{K} \in H^{m}(K) \text {, for all } K \in \mathcal{T}\right\}
$$

$\mathcal{E}_{h}$ is the set of all edges $e$ of elements $K$ of $\mathcal{T}$, its subset of all elements that are not contained in $\partial \Omega$ is denoted by $\mathcal{E}_{h}^{0}$, and its subset of all boundary elements is denoted by $\mathcal{E}_{h}^{b}=\mathcal{E}_{h} \backslash \mathcal{E}_{h}^{0}$. $\mathcal{E}_{h}^{s}$ is the set of boundary elements that are on $S$. For each $e \in \mathcal{E}_{h}, \boldsymbol{b}_{e}$ is the midpoint of $e$. Finally, let $\mathcal{N}_{h}$ be the set of vertices of the element of $\mathcal{T}$, and $\mathcal{N}_{h}^{0}$ the subset of those that are inside $\Omega$ and $\mathcal{N}_{h}^{b}=\mathcal{N}_{h} \backslash \mathcal{N}_{h}^{0}$ denote the set of vertices of $\mathcal{T}$ on the boundary. In the analysis of the problem, we will require jump, and average value of quantities. So let $K^{+}$and $K^{-}$be two adjacent elements of $\mathcal{T}$, and $e=\partial K^{+} \cap \partial K^{-}$. For scalar, vector, and matrix-valued functions $q \in H^{1}(\Omega, \mathcal{T}), \boldsymbol{v} \in \boldsymbol{H}^{1}(\Omega, \mathcal{T})$, and $\underline{\boldsymbol{\tau}} \in\left[H^{1}(\Omega, \mathcal{T})\right]^{2 \times 2}$, we define the following averages operator at the edge $e=\partial K^{+} \cap \partial K^{-}$:

$$
\{q\}=\frac{1}{2}\left(q^{+}+q^{-}\right), \quad\{\boldsymbol{v}\}=\frac{1}{2}\left(\boldsymbol{v}^{+}+\boldsymbol{v}^{-}\right), \quad\{\underline{\boldsymbol{\tau}}\}=\frac{1}{2}\left(\underline{\boldsymbol{\tau}}^{+}+\underline{\boldsymbol{\tau}}^{-}\right) .
$$

Similarly, the jumps at the edge $e=\partial K^{+} \cap \partial K^{-}$are given by

$$
[q]=q^{+} \boldsymbol{n}_{K^{+}}+q^{-} \boldsymbol{n}_{K^{-}},[\boldsymbol{v}]=\boldsymbol{v}^{+} \cdot \boldsymbol{n}_{K^{+}}+\boldsymbol{v}^{-} \cdot \boldsymbol{n}_{K^{-}}, \quad[\underline{\boldsymbol{v}}]=\boldsymbol{v}^{+} \otimes \boldsymbol{n}_{K^{+}}+\boldsymbol{v}^{-} \otimes \boldsymbol{n}_{K^{-}},
$$

and

$$
[\underline{\tau}]=\underline{\tau}^{+}+\underline{\tau}^{-}
$$

where for two vectors in Cartesian coordinates $\boldsymbol{a}=\left(a_{i}\right)$, and $\boldsymbol{b}=\left(b_{j}\right)$, we have $\boldsymbol{a} \otimes \boldsymbol{b}=a_{i} b_{j}$. If $e$ is an edge of element $K$ that lies on $\partial \Omega$, then the averages and jumps are defined by

$$
\{q\}=q, \quad\{\boldsymbol{v}\}=\boldsymbol{v}, \quad\{\underline{\boldsymbol{\tau}}\}=\underline{\boldsymbol{\tau}}
$$

and

$$
[q]=q \boldsymbol{n},[\boldsymbol{v}]=\boldsymbol{v} \cdot \boldsymbol{n},[\underline{\boldsymbol{v}}]=\boldsymbol{v} \otimes \boldsymbol{n},[\underline{\boldsymbol{\tau}}]=\underline{\boldsymbol{\tau}} \boldsymbol{n}
$$

where $\boldsymbol{n}$ denotes the unit outward normal vector to $\partial \Omega$. For each $K \in \mathcal{T}$, and for each nonnegative integer $k, \mathcal{P}_{k}(K)$ is the space of restrictions to $K$ of polynomials with 2 variables with degree less than or equal to $k$.

In all that follows, $\boldsymbol{c}$ is a generic constant independent of $h$. The discrete space of pressure $M_{h}$ consists of piecewise constant functions, namely

$$
M_{h}=\left\{q_{h} \in M: \forall K \in \mathcal{T},\left.q_{h}\right|_{K} \in \mathcal{P}_{0}(K)\right\}
$$

Its local interpolation operator is the orthogonal projection operator $\Pi_{K}^{h}$ from $M$ onto $M_{h}$ associated with the scalar product of $L^{2}(\Omega)$. That is

$$
\text { for all }\left(K, q, q_{h}\right) \in \mathcal{T} \times M \times M_{h}, \quad \int_{K} q_{h}\left(\Pi_{K}^{h} q-q\right)=0 .
$$

Assuming that $\mathcal{T}$ is a regular family of triangulations, and for any real number $s \in[0,1]$, there exists a constant $\boldsymbol{c}$, independent of $h$ such that [35]:

$$
\text { for all } q \in H^{s}(\Omega) \cap M,\left\|q-\Pi_{K}^{h} q\right\|_{K} \leq c h_{K}^{s}|q|_{s, K} \text {. }
$$


The global interpolation operator for the pressure is $\Pi_{h}: M \longrightarrow M_{h}$ and defined as follows

$$
\left.\left(\Pi_{h} q\right)\right|_{K}=\Pi_{K}^{h} q_{K} \text { for all }(K, q) \in \mathcal{T} \times M,
$$

with $q_{K}$ the restriction of $q$ on $K$. For approximating the velocity, one considers the element known in the mathematical circle as Crouzeix-Raviart elements [10]

$$
\mathcal{V}_{h}=\left\{\boldsymbol{v}_{h} \in \boldsymbol{L}^{2}(\Omega): \forall K \in \mathcal{T},\left.\boldsymbol{v}_{h}\right|_{K} \in \mathcal{P}_{1}(K), \forall e \in \mathcal{E}_{h}^{0}, \boldsymbol{v}_{h} \text { is continuous at } \boldsymbol{b}_{e}\right\}
$$

Its degree of freedom are located at $\boldsymbol{b}_{e}$ for all $e \in \mathcal{E}_{h}$, and its interpolation operator is the CrouzeixRaviart interpolant $I_{K}^{h}: \boldsymbol{H}^{1}(K) \longmapsto \mathcal{V}_{h}$ given as follows

$$
\left(I_{K}^{h} \boldsymbol{v}\right)\left(\boldsymbol{b}_{e}\right)=\frac{1}{h_{e}} \int_{e} \boldsymbol{v} d s \quad \text { for all }(\boldsymbol{v}, e) \in \boldsymbol{H}^{1}(K) \times \mathcal{E}_{K},
$$

where $\mathcal{E}_{K}$ is the set of three edges of $K$, and $h_{e}$ is the length of $e$. By the midpoint rule, one can also define $I_{K}^{h}$ with the equivalent condition

$$
\int_{e} I_{K}^{h} \boldsymbol{v} d s=\int_{e} \boldsymbol{v} d s \text { for all } e \in \mathcal{E}_{K} .
$$

The following interpolation error are valid for $I_{K}^{h}$ (see [10])

$$
\begin{aligned}
& \text { for all }(\boldsymbol{v}, K) \in \boldsymbol{H}^{1}(K) \times \mathcal{T}, \quad\left\|\boldsymbol{v}-I_{K}^{h} \boldsymbol{v}\right\|_{K}+h_{K}\left|\boldsymbol{v}-I_{K}^{h} \boldsymbol{v}\right|_{K} \leq \boldsymbol{c} h_{K}|\boldsymbol{v}|_{1, K}, \\
& \text { for all }(\boldsymbol{v}, K) \in \boldsymbol{H}^{2}(K) \times \mathcal{T}, \quad\left\|\boldsymbol{v}-I_{K}^{h} \boldsymbol{v}\right\|_{K}+h_{K}\left|\boldsymbol{v}-I_{K}^{h} \boldsymbol{v}\right|_{K} \leq \boldsymbol{c} h_{K}^{2}|\boldsymbol{v}|_{2, K} .
\end{aligned}
$$

The global interpolation operator for the velocity $I^{h}: \boldsymbol{V} \longrightarrow \boldsymbol{V}_{h}$ is defined as follows

$$
\left.\left(I^{h} \boldsymbol{v}\right)\right|_{K}=I_{K}^{h} \boldsymbol{v}_{K} \text { for all }(K, \boldsymbol{v}) \in \mathcal{T} \times \boldsymbol{V},
$$

with $\boldsymbol{v}_{K}$ the restriction of $\boldsymbol{v}$ on $K$. Finally to approximate the space $V$, we set

$$
\boldsymbol{V}_{h}=\left\{\boldsymbol{v}_{h} \in \mathcal{V}_{h}: \quad \forall e \in \mathcal{E}_{h}^{b},\left.\boldsymbol{v}_{h}\right|_{\Gamma \cap e}\left(\boldsymbol{b}_{e}\right)=0,\left.\quad \boldsymbol{v}_{h} \cdot \boldsymbol{n}\right|_{S \cap e}\left(\boldsymbol{b}_{e}\right)=0\right\} .
$$

We also note due to the midpoint rule that

$$
\boldsymbol{v} \in \boldsymbol{V}_{h} \text { implies } \int_{e}[\boldsymbol{v}] d s=0 \quad \forall e \in \mathcal{E}_{h} .
$$

We equip $\boldsymbol{V}_{h}$ with the broken semi-norm

$$
\left.\|\boldsymbol{v}\|\right|^{2}=\sum_{K \in \mathcal{T}}|\boldsymbol{v}|_{1, K}^{2}
$$

The continuity requirement at the center of each element of $\mathcal{E}_{h}^{0}$, together with the boundary conditions imply that this is a norm on $\boldsymbol{V}_{h}$. It should be noted that Poincaré-Friedrichs inequality is valid in $\boldsymbol{V}_{h}$, and reads (see $[30,31,36]$ ): there exists a constant $\boldsymbol{c}$ independent of the triangulation $\mathcal{T}$ such

$$
\text { for all } \boldsymbol{v}_{h} \in \boldsymbol{V}_{h}, \int_{\Omega}\left|\boldsymbol{v}_{h}\right|^{2} d x \leq \boldsymbol{c} \sum_{K \in \mathcal{T}} \int_{K}\left|\nabla \boldsymbol{v}_{h}\right|^{2} d x .
$$

Because we are dealing with second order operator in space, the following integration by parts formula (obtained by re-arranging terms) will be useful: for $\boldsymbol{v} \in \boldsymbol{H}^{2}(\Omega ; \mathcal{T})$ and $\boldsymbol{w} \in \boldsymbol{H}^{1}(\Omega ; \mathcal{T})$, it holds that

$$
\begin{aligned}
& \sum_{K} \int_{K}(\nabla \boldsymbol{v}: \nabla \boldsymbol{w}+\Delta \boldsymbol{v} \cdot \boldsymbol{w}) d x=\sum_{e \in \mathcal{E}_{h}} \int_{e}\{\nabla \boldsymbol{v}\}:[\underline{\boldsymbol{w}}] d s \\
& +\sum_{e \in \mathcal{E}_{h}^{0}} \int_{e}[\nabla \boldsymbol{v}] \cdot\{\boldsymbol{w}\} d s .
\end{aligned}
$$




\section{Stokes system: A Priori Error Estimate}

In Subsection 3.1, we formulate the finite element problem associated with (2.17), and quickly indicate how its solvability is obtained. We also introduce an equivalent three field formulation following Proposition 2.1. Subsection 3.2, and Subsection 3.3 are concerned about the derivation of the a priori error estimate for the velocity and pressure respectively, and constitute the main contributions of this section.

\subsection{Some preliminaries}

With the space $\boldsymbol{V}_{h}, M_{h}$ in place, we then approximate (2.17) as follows:

$$
\left\{\begin{array}{l}
\operatorname{Problem}(\mathbf{P h}) \\
\text { Find }\left(\boldsymbol{u}_{h}, p_{h}\right) \in V_{h} \times M_{h} \text { such that } \\
a_{h}\left(\boldsymbol{u}_{h}, \boldsymbol{v}_{h}-\boldsymbol{u}_{h}\right)+b_{h}\left(\boldsymbol{v}_{h}-\boldsymbol{u}_{h}, p_{h}\right)+j_{h}\left(\boldsymbol{v}_{h}\right)-j_{h}\left(\boldsymbol{u}_{h}\right) \geq \ell_{h}\left(\boldsymbol{v}_{h}-\boldsymbol{u}_{h}\right) \\
b_{h}\left(\boldsymbol{u}_{h}, q_{h}\right)=0 \\
\text { for all }\left(q_{h}, \boldsymbol{v}_{h}\right) \in M_{h} \times \boldsymbol{V}_{h}
\end{array}\right.
$$

with;

$$
\begin{aligned}
a_{h}(\boldsymbol{v}, \boldsymbol{w}) & =\nu \sum_{K \in \mathcal{T}} \int_{K} \nabla_{h} \boldsymbol{v}: \nabla_{h} \boldsymbol{w} d x+\nu \sum_{e \in \mathcal{E}_{h}^{s}}\left(\kappa \boldsymbol{v}_{\boldsymbol{\tau}}, \boldsymbol{w}_{\boldsymbol{\tau}}\right)_{e}, \quad \ell_{h}(\boldsymbol{v})=\sum_{K \in \mathcal{T}} \int_{K} \boldsymbol{f} \cdot \boldsymbol{v} d x, \\
b_{h}(\boldsymbol{v}, q) & =-\sum_{K} \int_{K} q \operatorname{div}_{h} \boldsymbol{v} d x, \quad j_{h}(\boldsymbol{v})=\sum_{e \in \mathcal{E}_{h}^{s}} \int_{e} g\left|\boldsymbol{v}_{\boldsymbol{\tau}}\right| d s,
\end{aligned}
$$

where $\nabla_{h}$ is the element-wise gradient, while $\operatorname{div}_{h}$ is the divergence evaluated element-wise. One sees that the bilinear form $a_{h}(\cdot, \cdot)$ coincides with $a(\cdot, \cdot)$ on $\boldsymbol{H}^{1}(\Omega) \times \boldsymbol{H}^{1}(\Omega)$, but differ on $\boldsymbol{V}_{h} \times \boldsymbol{V}_{h}$. The same conclusion can be drawn about $b_{h}(\cdot, \cdot)$ and $b(\cdot, \cdot)$. It follows from abstract approximation theory of mixed variational problems (see for instance $[8,23,24,27]$ ) that reasonable error estimates can be obtained for problem (3.1) if the bilinear form $a_{h}(\cdot, \cdot)$ is $\boldsymbol{V}_{h}$ elliptic, the bilinear form $b_{h}(\cdot, \cdot)$ is inf-sup stable, both uniform with respect to $h$, and $j_{h}(\cdot)$ is lower semi-continuous on $\boldsymbol{V}_{h}$.

Crouzeix and Raviart [10] have shown that with the spaces $\boldsymbol{V}_{h}$ and $M_{h}$ introduced, the bilinear form $b_{h}(\cdot, \cdot)$ is inf-sup stable in the sense that there exists a constant $\beta_{1}$ independent of the triangulation $\mathcal{T}$ such that

$$
\text { for all } q_{h} \in M_{h}, \beta_{1}\left\|q_{h}\right\| \leq \sup _{\boldsymbol{v}_{h} \in \boldsymbol{V}_{h}} \frac{b_{h}\left(\boldsymbol{v}_{h}, q_{h}\right)}{\|\| \boldsymbol{v}_{h} \mid \|} .
$$

Remark 3.1 (a) It is worth mentioning at this point that B. Lamichhane in [37] has shown that (3.3) holds if the pressure is discretized by piece-wise linear function, and $\boldsymbol{V}_{h}$ is as defined.

(b) The discrete kernel of the bilinear form $b_{h}(\cdot, \cdot)$ is defined as follows

$$
\mathcal{Z}_{h}=\left\{\boldsymbol{v}_{h} \in \boldsymbol{V}_{h}: \text { for all } q_{h} \in M_{h}, b_{h}\left(\boldsymbol{v}_{h}, q_{h}\right)=0\right\},
$$

and characterized by

$$
\mathcal{Z}_{h}=\left\{\boldsymbol{v} \in \boldsymbol{V}_{h}: \text { for all } e \in \mathcal{E}_{h}^{0},[\boldsymbol{v}]_{e}=0\right\}
$$


Thus, one observes that if $\left(\boldsymbol{u}_{h}, p_{h}\right) \in \boldsymbol{V}_{h} \times M_{h}$ is the solution of Problem (Ph), then $\operatorname{div} \boldsymbol{u}_{h}=$ 0 on each element $K \in \mathcal{T}$.

From the definition of both $a_{h}(\cdot, \cdot)$, and the mesh dependent norm ||$|\cdot|||$, it holds that

$$
\text { for all } \boldsymbol{v}_{h} \in \boldsymbol{V}_{h}, a_{h}\left(\boldsymbol{v}_{h}, \boldsymbol{v}_{h}\right)=\nu\|\| \boldsymbol{v}_{h} \mid\left\|^{2}+\nu\right\| \kappa^{1 / 2} \boldsymbol{v}_{h, \boldsymbol{\tau}}\left\|_{S}^{2} \geq \nu\right\|\left\|\boldsymbol{v}_{h}\right\| \|^{2},
$$

from which we deduce that $a_{h}(\cdot, \cdot)$ is $\boldsymbol{V}_{h}$-elliptic, which together with the continuity of $a_{h}(\cdot, \cdot)$, (3.3), the convexity and lower semi continuity of $j_{h}$ in $\boldsymbol{V}_{h}$, the continuity of $\ell_{h}(\cdot)$ in $\boldsymbol{V}_{h}$, one claims the following

Proposition 3.1 If $\boldsymbol{f}$ is an element of $\boldsymbol{L}^{2}(\Omega)$, and $g \in L^{\infty}(S)$, then the variational problem (3.1) has a unique solution $\left(\boldsymbol{u}_{h}, p_{h}\right) \in \boldsymbol{V}_{h} \times M_{h}$, which satisfies the estimate

$$
\left\|\boldsymbol{u}_{h} \mid\right\| \leq \frac{\boldsymbol{c}}{\nu}\|\boldsymbol{f}\| \quad\left\|p_{h}\right\| \leq \boldsymbol{c}\|\boldsymbol{f}\|+\boldsymbol{c} \frac{\kappa_{0}}{\nu}\|\boldsymbol{f}\| .
$$

The bounds (3.5) are obtained by direct application of the coercivity of $a_{h}(\cdot, \cdot)$ and inf-sup condition on $b_{h}(\cdot, \cdot)$.

Before we present the a priori error estimate for the velocity, we introduce the following interpolation which will play a crucial role in this study. Let $(\boldsymbol{u}, p)$ the unique solution of $(2.17)$, then there exist a unique couple $\left(\mathcal{K}_{h}, J_{h}\right)$,

$$
\left(\mathcal{K}_{h}, J_{h}\right): \boldsymbol{V} \times M \rightarrow \boldsymbol{V}_{h} \times M_{h}
$$

such that

$$
\left\{\begin{array}{l}
\text { for all }\left(\boldsymbol{v}_{h}, q_{h}\right) \in \boldsymbol{V}_{h} \times M_{h} \\
a_{h}\left(\mathcal{K}_{h} \boldsymbol{u}-\boldsymbol{u}, \boldsymbol{v}_{h}\right)+b_{h}\left(\boldsymbol{v}_{h}, J_{h} p-p\right)=0 \\
b_{h}\left(\mathcal{K}_{h} \boldsymbol{u}, q_{h}\right)=0
\end{array}\right.
$$

Furthermore the following properties are valid

$$
\frac{1}{h}\left\|\boldsymbol{u}-\mathcal{K}_{h} \boldsymbol{u}\right\|+\left\|\boldsymbol{u}-\mathcal{K}_{h} \boldsymbol{u}\right\| \mid \leq \boldsymbol{c h}\|\boldsymbol{u}\|_{2},\left\|J_{h} p-p\right\| \leq \boldsymbol{c h}\|p\|_{1}
$$

It should be noted that the existence of $\left(\mathcal{K}_{h}, J_{h}\right)$ is due thanks to the properties of $a_{h}(\cdot, \cdot)$ and $b_{h}(\cdot, \cdot)$, while the error estimate $(3.7)$ are derived with the help of the triangle inequality, $(2.52)$, (2.50), and the properties of $a_{h}(\cdot, \cdot)$ and $b_{h}(\cdot, \cdot)$.

\subsection{A Priori Error on the velocity}

The convergence result for the velocity can be stated as follows.

Proposition 3.2 Let $(\boldsymbol{f}, g) \in \boldsymbol{L}^{2}(\Omega) \times L^{\infty}(S) \cap H^{1}(S)$. Let $(\boldsymbol{u}, p)$ and $\left(\boldsymbol{u}_{h}, p_{h}\right)$ be solutions of 
(2.17) and (3.1) respectively. If $(\boldsymbol{u}, p)$ belong to $\boldsymbol{H}^{2}(\Omega) \times H^{1}(\Omega)$, then

$$
\begin{aligned}
\|\| \boldsymbol{u}-\boldsymbol{u}_{h}\|\| \leq & \boldsymbol{c}\left\|\mathcal{K}_{h} \boldsymbol{u}-\boldsymbol{u}\right\|+\boldsymbol{c}\left(\sum_{e \in \mathcal{E}_{h}^{s}}\left(1+h_{e}^{2}\right)\|g\|_{1, e}^{2}\right)^{1 / 4}\left(\sum_{e \in \mathcal{E}_{h}^{s}}\left\|\left(\mathcal{K}_{h} \boldsymbol{u}\right)_{\boldsymbol{\tau}}-\boldsymbol{u}_{\boldsymbol{\tau}}\right\|_{e}^{2}\right)^{1 / 4} \\
& +\boldsymbol{c h}\left(\|\boldsymbol{u}\|_{2}^{2}+\|p\|_{1}^{2}+\sum_{e \in \mathcal{E}_{h}}\|\kappa\|_{1, e}^{2}\right)^{1 / 2} \\
& +\boldsymbol{c h}^{1 / 2}\left[h\left(\sum_{e \in \mathcal{E}_{h}^{s}}\|g\|_{1, e}^{2}\right)^{1 / 2}+\left(\sum_{e \in \mathcal{E}_{h}^{s}}\left(h_{e}^{2}+1\right)\|g\|_{1, e}^{2}\right)^{1 / 2}\right]
\end{aligned}
$$

where $\boldsymbol{c}$ is a positive constant independent of $h$.

Remark 3.2 The analysis here is based on the assumption that $(\boldsymbol{u}, p) \in \boldsymbol{H}^{2}(\Omega) \times H^{1}(\Omega)$. The proof uses Babuska-Brezzi's conditions for stability or convergence of mixed problems, but the new twist in our analysis is the introduction of the enrichment map (initially defined by S.C. Brenner in [38]) to put together the continuous and finite element problems. The error estimate obtained is not optimal with respect to the polynomial approximating the velocity, and this lack of optimality is not surprising for this kind of problem (see [19, 20, 28]). It should be pointed out that the lack of consistency of the discrete problem (3.1), combined with the fact that $\boldsymbol{V}_{h}$ is not a subset of $\boldsymbol{V}$, and the presence of non-differentiable functional $j_{h}(\cdot)$ in the variational formulation make the analysis of the error not a trivial task. Also of great importance in the analysis below is the utilization of equivalent formulation (2.22).

Proof of Proposition 3.2. It is divided in many steps.

\section{Step 1: Some preliminaries}

We first introduce/recall the following trace and inverse inequalities (see [39]):

$$
\begin{aligned}
& \text { for all }(\boldsymbol{v}, K) \in \boldsymbol{H}^{1}(K) \times \mathcal{T}, \quad\|\boldsymbol{v}\|_{e} \leq \boldsymbol{c}\left(h_{e}^{-1 / 2}\|\boldsymbol{v}\|_{K}+h_{e}^{1 / 2}|\boldsymbol{v}|_{1, K}\right), \\
& \text { for all } \boldsymbol{v}_{h} \in \boldsymbol{V}_{h}, \sum_{e \in \mathcal{E}_{h}} \frac{1}{h_{e}}\left\|\left[\boldsymbol{v}_{h}\right]\right\|_{e}^{2} \leq \boldsymbol{c}\left\|\boldsymbol{v}_{h}\right\| \|^{2} \text {, } \\
& \text { for all }\left(\boldsymbol{v}_{h}, K\right) \in \boldsymbol{V}_{h} \times \mathcal{T},\left\|\boldsymbol{v}_{h}\right\|_{e} \leq \boldsymbol{c h}-1 / 2\left\|\boldsymbol{v}_{h}\right\|_{K} \text {, } \\
& \text { for all }\left(\boldsymbol{v}_{h}, K\right) \in \boldsymbol{V}_{h} \times \mathcal{T},\left|\boldsymbol{v}_{h}\right|_{1, K} \leq \boldsymbol{c} h_{K}^{-1}\left\|\boldsymbol{v}_{h}\right\|_{K} \text {. }
\end{aligned}
$$

We will require the conforming finite element space $\boldsymbol{V}_{h}^{c}$ given by

$$
\boldsymbol{V}_{h}^{c}=\boldsymbol{V}_{h} \cap \boldsymbol{V}
$$

We consider the smoothing map $E_{h}: \boldsymbol{V}_{h} \longrightarrow \boldsymbol{V}_{h}^{c}$ introduced first by S.C Brenner [38], defined as follows: For $\boldsymbol{v}_{h} \in \boldsymbol{V}_{h}$, we take $E_{h} \boldsymbol{v}_{h}$ such that;

$$
\left\{\begin{array}{l}
E_{h} \boldsymbol{v}_{h}(\boldsymbol{a})=\mathbf{0}, \text { for all vertices } \boldsymbol{a} \in \mathcal{N}_{h}^{b} \cap \Gamma \\
\left(E_{h} \boldsymbol{v}_{h}\right) \cdot \boldsymbol{n} \mid \boldsymbol{a}=\mathbf{0} \text { for all vertices } \boldsymbol{a} \in \mathcal{N}_{h}^{b} \cap S, \\
\left(E_{h} \boldsymbol{v}_{h}\right) \cdot \boldsymbol{\tau}\left|\boldsymbol{a}=\boldsymbol{v}_{h} \cdot \boldsymbol{\tau}\right| \boldsymbol{a} \text { for all vertices } \boldsymbol{a} \in \mathcal{N}_{h}^{b} \cap S,
\end{array}\right.
$$

Next

$$
\left(E_{h} \boldsymbol{v}_{h}\right)(\boldsymbol{a})=\frac{1}{\left|\mathcal{T}_{\boldsymbol{a}}\right|} \sum_{K \in \mathcal{T}_{\boldsymbol{a}}} \boldsymbol{v}_{h}(\boldsymbol{a}), \text { for all vertices } \boldsymbol{a} \in \mathcal{N}_{h}^{0},
$$


where $\mathcal{T}_{\boldsymbol{a}}$ is the set of triangles in $\mathcal{T}$ that share the vertex $\boldsymbol{a}$, and $\left|\mathcal{T}_{\boldsymbol{a}}\right|$ is the number of elements of $\mathcal{T}_{\boldsymbol{a}}$.

Remark 3.3 We observe from the definitions of both $\boldsymbol{V}_{h}$ and $E_{h}$ that $E_{h} \boldsymbol{v}_{h}$ belongs to $\boldsymbol{V}_{h}^{c}$.

The following error estimates on $E_{h}$ are important in our analysis ([38]).

$$
\begin{array}{r}
\text { for all } \boldsymbol{v}_{h} \in \boldsymbol{V}_{h}, \sum_{K \in \mathcal{T}} h_{K}^{-2}\left\|E_{h} \boldsymbol{v}_{h}-\boldsymbol{v}_{h}\right\|_{L^{2}(K)}^{2} \leq \boldsymbol{c} \sum_{e \in \mathcal{E}_{h}} \frac{1}{h_{e}}\left\|\left[\boldsymbol{v}_{h}\right]\right\|_{L^{2}(e)}^{2}, \\
\text { for all } \boldsymbol{v}_{h} \in \boldsymbol{V}_{h}, \sum_{K \in \mathcal{T}}\left|E_{h} \boldsymbol{v}_{h}-\boldsymbol{v}_{h}\right|_{1, K}^{2} \leq \boldsymbol{c} \sum_{e \in \mathcal{E}_{h}} \frac{1}{h_{e}}\left\|\left[\boldsymbol{v}_{h}\right]\right\|_{L^{2}(e)}^{2}, \\
\text { for all } \boldsymbol{v}_{h} \in \boldsymbol{V}_{h},\left|E_{h} \boldsymbol{v}_{h}\right|_{1}^{2} \leq \boldsymbol{c} \sum_{e \in \mathcal{E}_{h}} \frac{1}{h_{e}}\left\|\left[\boldsymbol{v}_{h}\right]\right\|_{L^{2}(e)}^{2} .
\end{array}
$$

The following Sobolev inequality will be useful [40]

Lemma 3.1 Let $I \subset \mathbb{R}$, a bounded open subset, for any $v \in H^{1}(I)$, then

$$
\|v\|_{L^{\infty}(I)} \leq\left[|I|+|I|^{-1}\right]^{1 / 2}\|v\|_{1, I}
$$

\section{Step 2: Use of incompressibility condition .}

From the second equations of (3.1) and (2.17), we deduce that

$$
\text { for all } q_{h} \in M_{h}, \quad b_{h}\left(\boldsymbol{u}-\boldsymbol{u}_{h}, q_{h}\right)=0 .
$$

Step 3: Use of coercivity condition on $a_{h}(\cdot, \cdot)$.

We note that $\boldsymbol{V}_{h}$ is not a subset of $\boldsymbol{V}$, but by construction (see (3.12)) $\boldsymbol{V}_{h}^{c}$ is a subspace of $\boldsymbol{V}$. From the coercivity of $a_{h}(\cdot, \cdot)$ and the first equation in $(3.1)$, it holds that

$$
\begin{aligned}
\left.\nu\left\|\boldsymbol{u}_{h}-\mathcal{K}_{h} \boldsymbol{u}\right\|\right|^{2} \leq & a_{h}\left(\boldsymbol{u}_{h}-\mathcal{K}_{h} \boldsymbol{u}, \boldsymbol{u}_{h}-\mathcal{K}_{h} \boldsymbol{u}\right) \\
= & a_{h}\left(\boldsymbol{u}_{h}, \boldsymbol{u}_{h}-\mathcal{K}_{h} \boldsymbol{u}\right)-a_{h}\left(\mathcal{K}_{h} \boldsymbol{u}, \boldsymbol{u}_{h}-\mathcal{K}_{h} \boldsymbol{u}\right) \\
\leq & b_{h}\left(\mathcal{K}_{h} \boldsymbol{u}-\boldsymbol{u}_{h}, p_{h}\right)+j_{h}\left(\mathcal{K}_{h} \boldsymbol{u}\right)-j_{h}\left(\boldsymbol{u}_{h}\right)-\ell_{h}\left(\mathcal{K}_{h} \boldsymbol{u}-\boldsymbol{u}_{h}\right)-a_{h}\left(\mathcal{K}_{h} \boldsymbol{u}, \boldsymbol{u}_{h}-\mathcal{K}_{h} \boldsymbol{u}\right) \\
= & b_{h}\left(\mathcal{K}_{h} \boldsymbol{u}-\boldsymbol{u}_{h}, p_{h}\right)-\ell\left(\mathcal{K}_{h} \boldsymbol{u}-\boldsymbol{u}_{h}-E_{h}\left(\mathcal{K}_{h} \boldsymbol{u}-\boldsymbol{u}_{h}\right)\right)-a_{h}\left(\mathcal{K}_{h} \boldsymbol{u}-\boldsymbol{u}, \boldsymbol{u}_{h}-\mathcal{K}_{h} \boldsymbol{u}\right) \\
& -a_{h}\left(\boldsymbol{u}, \boldsymbol{u}_{h}-\mathcal{K}_{h} \boldsymbol{u}\right)+j_{h}\left(\mathcal{K}_{h} \boldsymbol{u}\right)-j_{h}\left(\boldsymbol{u}_{h}\right)-\ell\left(E_{h}\left(\mathcal{K}_{h} \boldsymbol{u}-\boldsymbol{u}_{h}\right)\right) .
\end{aligned}
$$

Using (3.18), the second equations in (3.6) and (2.17), we deduce that

$$
\text { for all } q_{h} \in M_{h}, \quad b_{h}\left(\mathcal{K}_{h} \boldsymbol{u}-\boldsymbol{u}_{h}, q_{h}\right)=b_{h}\left(\mathcal{K}_{h} \boldsymbol{u}-\boldsymbol{u}, q_{h}\right)+b_{h}\left(\boldsymbol{u}-\boldsymbol{u}_{h}, q_{h}\right)=0 .
$$

Hence (3.19) is reduced to

$$
\begin{aligned}
\nu\left\|\boldsymbol{u}_{h}-\mathcal{K}_{h} \boldsymbol{u}\right\| \|^{2} \leq & -\ell\left(\mathcal{K}_{h} \boldsymbol{u}-\boldsymbol{u}_{h}-E_{h}\left(\mathcal{K}_{h} \boldsymbol{u}-\boldsymbol{u}_{h}\right)\right)-a_{h}\left(\mathcal{K}_{h} \boldsymbol{u}-\boldsymbol{u}, \boldsymbol{u}_{h}-\mathcal{K}_{h} \boldsymbol{u}\right) \\
& -a_{h}\left(\boldsymbol{u}, \boldsymbol{u}_{h}-\mathcal{K}_{h} \boldsymbol{u}\right)+j_{h}\left(\mathcal{K}_{h} \boldsymbol{u}\right)-j_{h}\left(\boldsymbol{u}_{h}\right)-\ell\left(E_{h}\left(\mathcal{K}_{h} \boldsymbol{u}-\boldsymbol{u}_{h}\right)\right) .
\end{aligned}
$$

We take $\boldsymbol{v}_{h}=\boldsymbol{u}_{h}-\mathcal{K}_{h} \boldsymbol{u}$ in (3.6) together with (3.20) we find

$$
-a_{h}\left(\mathcal{K}_{h} \boldsymbol{u}-\boldsymbol{u}, \boldsymbol{u}_{h}-\mathcal{K}_{h} \boldsymbol{u}\right)=b_{h}\left(\boldsymbol{u}_{h}-\mathcal{K}_{h} \boldsymbol{u}, J_{h} p-p\right)=b_{h}\left(\mathcal{K}_{h} \boldsymbol{u}-\boldsymbol{u}_{h}, p\right) .
$$


Hence (3.21) becomes

$$
\begin{aligned}
\nu\left\|\boldsymbol{u}_{h}-\mathcal{K}_{h} \boldsymbol{u} \mid\right\|^{2} \leq & -\ell\left(\mathcal{K}_{h} \boldsymbol{u}-\boldsymbol{u}_{h}-E_{h}\left(\mathcal{K}_{h} \boldsymbol{u}-\boldsymbol{u}_{h}\right)\right)+b_{h}\left(\mathcal{K}_{h} \boldsymbol{u}-\boldsymbol{u}_{h}, p\right) \\
& -a_{h}\left(\boldsymbol{u}, \boldsymbol{u}_{h}-\mathcal{K}_{h} \boldsymbol{u}\right)+j_{h}\left(\mathcal{K}_{h} \boldsymbol{u}\right)-j_{h}\left(\boldsymbol{u}_{h}\right)-\ell\left(E_{h}\left(\mathcal{K}_{h} \boldsymbol{u}-\boldsymbol{u}_{h}\right)\right)
\end{aligned}
$$

Now in the first equation of (2.22), setting $\boldsymbol{v}=E_{h}\left(\mathcal{K}_{h} \boldsymbol{u}-\boldsymbol{u}_{h}\right) \in \boldsymbol{V}_{h}^{c} \subset \boldsymbol{V}$, we find that

$$
\ell_{h}\left(E_{h}\left(\mathcal{K}_{h} \boldsymbol{u}-\boldsymbol{u}_{h}\right)\right)=a\left(\boldsymbol{u}, E_{h}\left(\mathcal{K}_{h} \boldsymbol{u}-\boldsymbol{u}_{h}\right)\right)+b\left(E_{h}\left(\mathcal{K}_{h} \boldsymbol{u}-\boldsymbol{u}_{h}\right), p\right)+\int_{S} g \boldsymbol{\alpha} \cdot\left(E_{\boldsymbol{\tau}, h}\left(\mathcal{K}_{h} \boldsymbol{u}-\boldsymbol{u}_{h}\right)\right),
$$

with the notation $E_{\boldsymbol{\tau}, h} \boldsymbol{v}_{h}=\left(E_{h} \boldsymbol{v}_{h}\right)_{\boldsymbol{\tau}}$. Inserting (3.24) in (3.23), it holds that

$$
\begin{aligned}
& \nu\left\|\boldsymbol{u}_{h}-\mathcal{K}_{h} \boldsymbol{u}\right\| \|^{2} \\
\leq & -\ell\left(\mathcal{K}_{h} \boldsymbol{u}-\boldsymbol{u}_{h}-E_{h}\left(\mathcal{K}_{h} \boldsymbol{u}-\boldsymbol{u}_{h}\right)\right)+b_{h}\left(\mathcal{K}_{h} \boldsymbol{u}-\boldsymbol{u}_{h}-E_{h}\left(\mathcal{K}_{h} \boldsymbol{u}-\boldsymbol{u}_{h}\right), p\right)+a_{h}\left(\boldsymbol{u}, \mathcal{K}_{h} \boldsymbol{u}-\boldsymbol{u}_{h}-E_{h}\left(\mathcal{K}_{h} \boldsymbol{u}-\boldsymbol{u}_{h}\right)\right) \\
& +j_{h}\left(\mathcal{K}_{h} \boldsymbol{u}\right)-j_{h}\left(\boldsymbol{u}_{h}\right)-\int_{S} g \boldsymbol{\alpha} \cdot\left(\mathcal{K}_{h} \boldsymbol{u}-\boldsymbol{u}_{h}\right)_{\boldsymbol{\tau}}-\int_{S} g \boldsymbol{\alpha} \cdot\left(E_{h}\left(\mathcal{K}_{h} \boldsymbol{u}-\boldsymbol{u}_{h}\right)-\left(\mathcal{K}_{h} \boldsymbol{u}-\boldsymbol{u}_{h}\right)\right)_{\boldsymbol{\tau}}
\end{aligned}
$$

We now want to estimate the expressions appearing on the right hand side of (3.25).

First, using the triangle inequality, the Cauchy-Shwarz inequality and the trace's inequality, we have

$$
\begin{aligned}
& j_{h}\left(\mathcal{K}_{h} \boldsymbol{u}\right)-j_{h}\left(\boldsymbol{u}_{h}\right)-\int_{S} g \boldsymbol{\alpha} \cdot\left(\mathcal{K}_{h} \boldsymbol{u}-\boldsymbol{u}_{h}\right)_{\boldsymbol{\tau}} \\
= & \sum_{e \in \mathcal{E}_{h}^{s}} \int_{e} g\left(\left|\left(\mathcal{K}_{h} \boldsymbol{u}\right)_{\boldsymbol{\tau}}\right|-\left|\left(\boldsymbol{u}_{h}\right)_{\boldsymbol{\tau}}\right|-\boldsymbol{\alpha} \cdot\left(\mathcal{K}_{h} \boldsymbol{u}\right)_{\boldsymbol{\tau}}+\boldsymbol{\alpha} \cdot\left(\boldsymbol{u}_{h}\right)_{\boldsymbol{\tau}}\right) \\
\leq & \sum_{e \in \mathcal{E}_{h}^{s}} \int_{e} g\left(\left|\left(\mathcal{K}_{h} \boldsymbol{u}\right)_{\boldsymbol{\tau}}\right|-\left|\left(\boldsymbol{u}_{h}\right)_{\boldsymbol{\tau}}\right|-\boldsymbol{\alpha} \cdot\left(\mathcal{K}_{h} \boldsymbol{u}\right)_{\boldsymbol{\tau}}+\left|\left(\boldsymbol{u}_{h}\right)_{\boldsymbol{\tau}}\right|\right) \quad(\text { using }|\boldsymbol{\alpha}| \leq 1) \\
= & \sum_{e \in \mathcal{E}_{h}^{s}} \int_{e} g\left(\left|\left(\mathcal{K}_{h} \boldsymbol{u}\right)_{\boldsymbol{\tau}}\right|-\boldsymbol{\alpha} \cdot\left(\mathcal{K}_{h} \boldsymbol{u}\right)_{\boldsymbol{\tau}}\right) \\
= & \sum_{e \in \mathcal{E}_{h}^{s}} \int_{e} g\left(\left|\left(\mathcal{K}_{h} \boldsymbol{u}\right)_{\boldsymbol{\tau}}\right|-\left|\boldsymbol{u}_{\boldsymbol{\tau}}\right|+\boldsymbol{\alpha} \cdot \boldsymbol{u}_{\boldsymbol{\tau}}-\boldsymbol{\alpha} \cdot\left(\mathcal{K}_{h} \boldsymbol{u}\right)_{\boldsymbol{\tau}}\right) \quad\left(\mathrm{using} \boldsymbol{\alpha} \cdot \boldsymbol{u}_{\boldsymbol{\tau}}=\left|\boldsymbol{u}_{\boldsymbol{\tau}}\right|\right) \\
\leq & \sum_{e \in \mathcal{E}_{h}^{s}} \int_{e} g\left|\left(\mathcal{K}_{h} \boldsymbol{u}\right)_{\boldsymbol{\tau}}-\boldsymbol{u}_{\boldsymbol{\tau}}\right|+\sum_{e \in \mathcal{E}_{h}^{s}} \int_{e} g \boldsymbol{\alpha} \cdot\left(\boldsymbol{u}_{\boldsymbol{\tau}}-\left(\mathcal{K}_{h} \boldsymbol{u}\right)_{\boldsymbol{\tau}}\right) \\
\leq & 2 \sum_{e \in \mathcal{E}_{h}^{s}} \int_{e} g\left|\left(\mathcal{K}_{h} \boldsymbol{u}\right)_{\boldsymbol{\tau}}-\boldsymbol{u}_{\boldsymbol{\tau}}\right| \\
\leq & 2 \sum_{e \in \mathcal{E}_{h}^{s}}\|g\|_{L^{\infty}(e)} h_{e}^{1 / 2}\left\|\left(\mathcal{K}_{h} \boldsymbol{u}\right)_{\boldsymbol{\tau}}-\boldsymbol{u}_{\boldsymbol{\tau}}\right\|_{e} \\
\leq & \boldsymbol{c}\left(\sum_{e \in \mathcal{E}_{h}^{s}}\left(1+h_{e}^{2}\right)\|g\|_{1, e}^{2}\right)^{1 / 2}\left(\sum_{e \in \mathcal{E}_{h}^{s}}\left\|\left(\mathcal{K}_{h} \boldsymbol{u}\right)_{\boldsymbol{\tau}}-\boldsymbol{u}_{\boldsymbol{\tau}}\right\|_{e}^{2}\right)^{1 / 2},
\end{aligned}
$$

where we have used Lemma 3.1. We let

$$
\boldsymbol{w}_{h}=\mathcal{K}_{h} \boldsymbol{u}-\boldsymbol{u}_{h}-E_{h}\left(\mathcal{K}_{h} \boldsymbol{u}-\boldsymbol{u}_{h}\right)
$$

and we would like to estimate $-\left(g \boldsymbol{\alpha}, \boldsymbol{w}_{\boldsymbol{\tau}, h}\right)_{S}$. For that purpose, the mean of a function $\phi$ over the edge $e$ is

$$
\Pi_{e}^{0} \phi=\frac{1}{h_{e}} \int_{e} \phi d s .
$$


From linearity we find that

$$
\begin{aligned}
-\left(g \boldsymbol{\alpha}, \boldsymbol{w}_{\boldsymbol{\tau}, h}\right)_{S} & =-\sum_{e \in \mathcal{E}_{h}^{s}} \int_{e}\left(g \boldsymbol{\alpha}, \boldsymbol{w}_{\boldsymbol{\tau}, h}\right)_{e} \\
& =\sum_{e \in \mathcal{E}_{h}^{s}}\left[\int_{e}\left(\Pi_{e}^{0} g-g\right) \boldsymbol{\alpha} \cdot \boldsymbol{w}_{\boldsymbol{\tau}, h}-\int_{e} \Pi_{e}^{0} g \boldsymbol{\alpha} \cdot \boldsymbol{w}_{\boldsymbol{\tau}, h}\right] .
\end{aligned}
$$

The following approximation result is important (see [40]).

Lemma 3.2 ( Poincare-Wirtinger's inequality). Let $I \subset \mathbb{R}$ be a bounded open subset, then

$$
\left\|\phi-\Pi_{I}^{0} \phi\right\|_{L^{\infty}(I)} \leq|I|^{1 / 2}\left\|\phi^{\prime}\right\|_{L^{2}(I)} \quad \text { for all } \phi \in H^{1}(I) .
$$

The first term on the right hand side of (3.27) is treated with the help of Cauchy-Schwarz inequality, Holder inequality, Lemma 3.2, (3.10), (3.15) and (3.9) as follows

$$
\begin{aligned}
\sum_{e \in \mathcal{E}_{h}^{s}} \int_{e}\left(\Pi_{e}^{0} g-g\right) \boldsymbol{\alpha} \cdot \boldsymbol{w}_{\boldsymbol{\tau}, h} & \leq \sum_{e \in \mathcal{E}_{h}^{s}}\left\|\Pi_{e}^{0} g-g\right\|_{L^{\infty}(e)}\|\boldsymbol{\alpha}\|_{e}\left\|\boldsymbol{w}_{\boldsymbol{\tau}, h}\right\|_{e} \\
& \leq \sum_{e \in \mathcal{E}_{h}^{s}}\left\|\Pi_{e}^{0} g-g\right\|_{L^{\infty}(e)} h_{e}^{1 / 2}\left\|\boldsymbol{w}_{\boldsymbol{\tau}, h}\right\|_{e} \\
& \leq \sum_{e \in \mathcal{E}_{h}^{s}} h_{e}\|g\|_{1, e}\left\|\boldsymbol{w}_{\boldsymbol{\tau}, h}\right\|_{e} \\
& \leq\left(\sum_{e \in \mathcal{E}_{h}^{s}}\|g\|_{1, e}^{2}\right)^{1 / 2}\left(\sum_{e \in \mathcal{E}_{h}^{s}} h_{e}^{2}\left\|\boldsymbol{w}_{\boldsymbol{\tau}, h}\right\|_{e}^{2}\right)^{1 / 2} \\
& \leq \boldsymbol{c}\left(\sum_{e \in \mathcal{E}_{h}^{s}}\|g\|_{1, e}^{2}\right)^{1 / 2}\left(\sum_{K \in \mathcal{T}}\left\|\boldsymbol{w}_{h}\right\|_{K}^{2}\right)^{1 / 2} h^{1 / 2} \\
& \leq \boldsymbol{c}\left(\sum_{e \in \mathcal{E}_{h}^{s}}\|g\|_{1, e}^{2}\right)^{1 / 2}\left(\sum_{e \in \mathcal{E}_{h}} \frac{1}{h_{e}}\left\|\left[\mathcal{K}_{h} \boldsymbol{u}-\boldsymbol{u}_{h}\right]\right\|_{e}^{2}\right)^{1 / 2} h^{3 / 2} \\
& \leq \boldsymbol{c h} h^{3 / 2}\left(\sum_{e \in \mathcal{E}_{h}^{s}}\|g\|_{1, e}^{2}\right)^{1 / 2}\left\|\mathcal{K}_{h} \boldsymbol{u}-\boldsymbol{u}_{h}\right\| \|
\end{aligned}
$$

Following the way we have obtained (3.28), and having in mind Lemma 3.1, we obtain

$$
\begin{aligned}
-\sum_{e \in \mathcal{E}_{h}^{s}} \int_{e} \Pi_{e}^{0} g \boldsymbol{\alpha} \cdot \boldsymbol{w}_{\boldsymbol{\tau}, h} & \leq \sum_{e \in \mathcal{E}_{h}^{s}}\|g\|_{L^{\infty}(e)}\|\boldsymbol{\alpha}\|_{e}\left\|\boldsymbol{w}_{\boldsymbol{\tau}, h}\right\|_{e} \\
& \leq \sum_{e \in \mathcal{E}_{h}^{s}}\left(h_{e}^{2}+1\right)^{1 / 2}\|g\|_{1, e}\left\|\boldsymbol{w}_{\boldsymbol{\tau}, h}\right\|_{e} \\
& \leq \boldsymbol{c h}^{1 / 2}\left(\sum_{e \in \mathcal{E}_{h}^{s}}\left(h_{e}^{2}+1\right)\|g\|_{1, e}^{2}\right)^{1 / 2}\|\| \mathcal{K}_{h} \boldsymbol{u}-\boldsymbol{u}_{h} \| .
\end{aligned}
$$


Returning to (3.27) with (3.29) and (3.28), we find

$$
\begin{aligned}
& -\left(g \boldsymbol{\alpha}, \boldsymbol{w}_{\boldsymbol{\tau}, h}\right)_{S} \\
\leq & \boldsymbol{c h}^{1 / 2}\left[h\left(\sum_{e \in \mathcal{E}_{h}^{s}}\|g\|_{1, e}^{2}\right)^{1 / 2}+\left(\sum_{e \in \mathcal{E}_{h}^{s}}\left(h_{e}^{2}+1\right)\|g\|_{1, e}^{2}\right)^{1 / 2}\right]\left\|\mathcal{K}_{h} \boldsymbol{u}-\boldsymbol{u}_{h}\right\| .
\end{aligned}
$$

The term $-\ell\left(\boldsymbol{w}_{h}\right)+b_{h}\left(\boldsymbol{w}_{h}, p\right)+a_{h}\left(\boldsymbol{u}, \boldsymbol{w}_{h}\right)$ is first re-written using (2.57), and the fact that $\boldsymbol{u} \in \boldsymbol{H}^{2}$, ( implies $[\nabla \boldsymbol{u}]=\mathbf{0},\{\nabla \boldsymbol{u}\}=\nabla \boldsymbol{u}$ ) as follows

$$
\begin{aligned}
a_{h}\left(\boldsymbol{u}, \boldsymbol{w}_{h}\right) & =\nu \sum_{K} \int_{K} \nabla_{h} \boldsymbol{u}: \nabla \boldsymbol{w}_{h}+\nu \sum_{e \in \mathcal{E}_{h}^{s}} \int_{e} \kappa \boldsymbol{u}_{\boldsymbol{\tau}} \cdot \boldsymbol{w}_{\boldsymbol{\tau}, h} \\
& =-\nu \sum_{K} \int_{K} \triangle \boldsymbol{u} \cdot \boldsymbol{w}_{h}+\nu \sum_{e \in \mathcal{E}_{h}} \int_{e}\{\nabla \boldsymbol{u}\}:\left[\underline{\left.\boldsymbol{w}_{h}\right]}+\nu \sum_{e \in \mathcal{E}_{h}^{0}} \int_{e}[\nabla \boldsymbol{u}] \cdot\left\{\boldsymbol{w}_{h}\right\}+\nu \sum_{e \in \mathcal{E}_{h}^{s}} \int_{e} \kappa \boldsymbol{u}_{\boldsymbol{\tau}} \cdot \boldsymbol{w}_{\boldsymbol{\tau}, h}\right. \\
& =-\nu \sum_{K} \int_{K} \Delta \boldsymbol{u} \cdot \boldsymbol{w}_{h}+\nu \sum_{e \in \mathcal{E}_{h}} \int_{e} \nabla \boldsymbol{u}:\left[\underline{\boldsymbol{w}_{h}}\right]+\nu \sum_{e \in \mathcal{E}_{h}^{s}} \int_{e} \kappa \boldsymbol{u}_{\boldsymbol{\tau}} \cdot \boldsymbol{w}_{\boldsymbol{\tau}, h} \\
& =-2 \nu \sum_{K} \int_{K} \operatorname{div} \boldsymbol{D}(\boldsymbol{u}) \cdot \boldsymbol{w}_{h}+\nu \sum_{e \in \mathcal{E}_{h}} \int_{e} \nabla \boldsymbol{u}:\left[\underline{\boldsymbol{w}}_{h}\right]+\nu \sum_{e \in \mathcal{E}_{h}^{s}} \int_{e} \kappa \boldsymbol{u}_{\boldsymbol{\tau}} \cdot \boldsymbol{w}_{\boldsymbol{\tau}, h}
\end{aligned}
$$

where (2.10) has been used. Again from the integration by parts (2.57) and taking $p \in H^{1}$, we find (having in mind $[p]=\mathbf{0},\{p\}=p$ ) that

$$
\begin{aligned}
b_{h}\left(\boldsymbol{w}_{h}, p\right) & =-\sum_{K} \int_{K} p \operatorname{div} \boldsymbol{w}_{h} \\
& =\sum_{K} \int_{K} \nabla p \cdot \boldsymbol{w}_{h}-\sum_{e \in \mathcal{E}_{h}} \int_{e}\{p\}\left[\boldsymbol{w}_{h}\right]-\sum_{e \in \mathcal{E}_{h}^{0}} \int_{e}[p] \cdot\left\{\boldsymbol{w}_{h}\right\} \\
& =\sum_{K} \int_{K} \nabla p \cdot \boldsymbol{w}_{h}-\sum_{e \in \mathcal{E}_{h}} \int_{e} p\left[\boldsymbol{w}_{h}\right] .
\end{aligned}
$$

Now, putting together (3.31) and (3.32) and using (1.1), one obtains

$$
\begin{aligned}
& -\ell\left(\boldsymbol{w}_{h}\right)+b_{h}\left(\boldsymbol{w}_{h}, p\right)+a_{h}\left(\boldsymbol{u}, \boldsymbol{w}_{h}\right) \\
= & \sum_{e \in \mathcal{E}_{h}} \int_{e}(-p \boldsymbol{I}+\nu \nabla \boldsymbol{u}):\left[\underline{\left.\boldsymbol{w}_{h}\right]}+\nu \sum_{e \in \mathcal{E}_{h}^{s}} \int_{e} \kappa \boldsymbol{u}_{\boldsymbol{\tau}} \cdot \boldsymbol{w}_{\boldsymbol{\tau}, h}\right. \\
= & \sum_{e \in \mathcal{E}_{h}} \int_{e}\left(\nu\left(\nabla \boldsymbol{u}-\Pi_{e}^{0} \nabla \boldsymbol{u}\right)-\left(p-\Pi_{e}^{0} p\right) \boldsymbol{I}\right):\left[\underline{\boldsymbol{w}_{h}}\right] d x+\nu \sum_{e \in \mathcal{E}_{h}^{s}} \int_{e}\left(\kappa-\Pi_{e}^{0} \kappa\right) \boldsymbol{w}_{\boldsymbol{\tau}, h} \cdot \boldsymbol{u}_{\boldsymbol{\tau}} \\
& +\nu \sum_{e \in \mathcal{E}_{h}^{s}} \int_{e} \Pi_{e}^{0} \kappa \boldsymbol{w}_{\boldsymbol{\tau}, h} \cdot\left(\boldsymbol{u}_{\boldsymbol{\tau}}-\Pi_{e}^{0} \boldsymbol{u}_{\boldsymbol{\tau}}\right) .
\end{aligned}
$$

We now treat each term on the right side of (3.33). It then follows from Cauchy-Schwarz inequality, (3.9), (3.10), (3.15), (3.16), and the approximation estimate that

$$
\begin{aligned}
& \sum_{e \in \mathcal{E}_{h}} \int_{e}\left(\nu\left(\nabla \boldsymbol{u}-\Pi_{e}^{0} \nabla \boldsymbol{u}\right)-\left(p-\Pi_{e}^{0} p\right) \boldsymbol{I}\right):\left[\underline{\boldsymbol{w}_{h}}\right] d x \\
= & \sum_{e \in \mathcal{E}_{h}} \int_{e}\left(\nu\left(\nabla \boldsymbol{u}-\Pi_{e}^{0} \nabla \boldsymbol{u}\right):\left[\underline{\boldsymbol{w}_{h}}\right] d x-\sum_{e \in \mathcal{E}_{h}} \int_{e}\left(p-\Pi_{e}^{0} p\right) \boldsymbol{I}\right):\left[\underline{\boldsymbol{w}_{h}}\right] d x \\
\leq & \left(\sum_{e \in \mathcal{E}_{h}} \nu h_{e}\left\|\nabla \boldsymbol{u}-\Pi_{e}^{0} \nabla \boldsymbol{u}\right\|_{e}^{2}\right)^{1 / 2}\left(\sum_{e \in \mathcal{E}_{h}} \frac{1}{h_{e}}\left\|\left[\underline{\boldsymbol{w}}_{h}\right]\right\|_{e}^{2}\right)^{1 / 2}+\left(\sum_{e \in \mathcal{E}_{h}} h_{e}\left\|p-\Pi_{e}^{0} p\right\|_{e}^{2}\right)^{1 / 2}\left(\sum_{e \in \mathcal{E}_{h}} \frac{1}{h_{e}}\left\|\left[\underline{\boldsymbol{w}_{h}}\right]\right\|_{e}^{2}\right)^{1 / 2} \\
\leq & \boldsymbol{c h}\left(\|\boldsymbol{u}\|_{2}+\|p\|_{1}\right)\left\|\mathcal{K}_{h} \boldsymbol{u}-\boldsymbol{u}_{h}\right\| \| .
\end{aligned}
$$


To bound the second term in the right hand side of (3.33), we use Cauchy-Schwarz inequality, Lemma 3.2, (3.10), (3.15), (3.9) as follows

$$
\begin{aligned}
& \sum_{e \in \mathcal{E}_{h}^{s}} \int_{e}\left(\kappa-\Pi_{e}^{0} \kappa\right) \boldsymbol{w}_{\boldsymbol{\tau}, h} \cdot \boldsymbol{u}_{\boldsymbol{\tau}} \\
\leq & \sum_{e \in \mathcal{E}_{h}^{s}}\left\|\kappa-\Pi_{e}^{0} \kappa\right\|_{L^{\infty}(e)}\left\|\boldsymbol{w}_{\boldsymbol{\tau}, h}\right\|_{e}\left\|\boldsymbol{u}_{\boldsymbol{\tau}}\right\|_{e} \\
\leq & \sum_{e \in \mathcal{E}_{h}^{s}} h_{e}^{1 / 2}\left\|\boldsymbol{w}_{\boldsymbol{\tau}, h}\right\|_{e}\|\kappa\|_{1, e}\left\|\boldsymbol{u}_{\boldsymbol{\tau}}\right\|_{e} \\
\leq & \left(\sum_{e \in \mathcal{E}_{h}^{s}} h_{e}\left\|\boldsymbol{w}_{\boldsymbol{\tau}, h}\right\|_{e}^{2}\right)^{1 / 2}\left(\sum_{e \in \mathcal{E}_{h}}\|\kappa\|_{1, e}^{2}\left\|\boldsymbol{u}_{\boldsymbol{\tau}}\right\|_{e}^{2}\right)^{1 / 2} \\
\leq & \boldsymbol{c}\left(\sum_{K \in \mathcal{T}}\left\|\boldsymbol{w}_{h}\right\|_{K}^{2}\right)^{1 / 2}\left(\sum_{e \in \mathcal{E}_{h}}\|\kappa\|_{1, e}^{2}\left\|\boldsymbol{u}_{\boldsymbol{\tau}}\right\|_{e}^{2}\right)^{1 / 2} \\
\leq & \boldsymbol{c h}\left(\sum_{e \in \mathcal{E}_{h}} \frac{1}{h_{e}}\left\|\left[\mathcal{K}_{h} \boldsymbol{u}-\boldsymbol{u}_{h}\right]\right\|_{e}^{2}\right)^{1 / 2}\left(\sum_{e \in \mathcal{E}_{h}}\|\kappa\|_{1, e}^{2}\left\|\boldsymbol{u}_{\boldsymbol{\tau}}\right\|_{e}^{2}\right)^{1 / 2} \\
\leq & \boldsymbol{c h}\left(\sum_{e \in \mathcal{E}_{h}}\|\kappa\|_{1, e}^{2}\left\|\boldsymbol{u}_{\boldsymbol{\tau}}\right\|_{e}^{2}\right)^{1 / 2}\|\| \mathcal{K}_{h} \boldsymbol{u}-\boldsymbol{u}_{h}\|\| .
\end{aligned}
$$

Finally, from the Cauchy-Schwarz inequality, Holder inequality, Lemma 3.1, (3.10), (3.15), (3.9) and approximation result, we get

$$
\begin{aligned}
\sum_{e \in \mathcal{E}_{h}^{s}} \int_{e} \Pi_{e}^{0} \kappa \boldsymbol{w}_{\boldsymbol{\tau}, h} \cdot\left(\boldsymbol{u}_{\boldsymbol{\tau}}-\Pi_{e}^{0} \boldsymbol{u}_{\boldsymbol{\tau}}\right) & \leq \sum_{e \in \mathcal{E}_{h}^{s}}\|\kappa\|_{L^{\infty}(e)}\left\|\boldsymbol{w}_{\boldsymbol{\tau}, h}\right\|_{e}\left\|\boldsymbol{u}_{\boldsymbol{\tau}}-\Pi_{e}^{0} \boldsymbol{u}_{\boldsymbol{\tau}}\right\|_{e} \\
& \leq \sum_{e \in \mathcal{E}_{h}^{s}}\left(h_{e}+h_{e}^{-1}\right)^{1 / 2}\|\kappa\|_{1, e}\left\|\boldsymbol{w}_{\boldsymbol{\tau}, h}\right\|_{e}\left\|\boldsymbol{u}_{\boldsymbol{\tau}}-\Pi_{e}^{0} \boldsymbol{u}_{\boldsymbol{\tau}}\right\|_{e} \\
& \leq\left(\sum_{e \in \mathcal{E}_{h}^{s}} \frac{1}{h_{e}}\left\|\boldsymbol{w}_{\boldsymbol{\tau}, h}\right\|_{e}^{2}\right)^{1 / 2}\left(\sum_{e \in \mathcal{E}_{h}^{s}}\left(h_{e}^{2}+1\right)\|\kappa\|_{1, e}^{2}\left\|\boldsymbol{u}_{\boldsymbol{\tau}}-\Pi_{e}^{0} \boldsymbol{u}_{\boldsymbol{\tau}}\right\|_{e}^{2}\right)^{1 / 2} \\
& \leq \boldsymbol{c}\left(\sum_{K \in \mathcal{T}} h_{K}^{-2}\left\|\boldsymbol{w}_{h}\right\|_{K}^{2}\right)^{1 / 2}\left(\sum_{e \in \mathcal{E}_{h}^{s}}\left(h_{e}^{2}+1\right)\|\kappa\|_{1, e}^{2}\left\|\boldsymbol{u}_{\boldsymbol{\tau}}-\Pi_{e}^{0} \boldsymbol{u}_{\boldsymbol{\tau}}\right\|_{e}^{2}\right)^{1 / 2} \\
& \leq \boldsymbol{c}\left(\sum_{e \in \mathcal{E}_{h}^{s}}\left(h_{e}^{2}+1\right)\|\kappa\|_{1, e}^{2}\left\|\boldsymbol{u}_{\boldsymbol{\tau}}-\Pi_{e}^{0} \boldsymbol{u}_{\boldsymbol{\tau}}\right\|_{e}^{2}\right)^{1 / 2}\left(\sum_{e \in \mathcal{E}_{h}} \frac{1}{h_{e}}\left\|\left[\mathcal{K}_{h} \boldsymbol{u}-\boldsymbol{u}_{h}\right]\right\|_{e}^{2}\right)^{1 / 2} \\
& \leq \boldsymbol{c h}\left(\sum_{e \in \mathcal{E}_{h}^{s}}\|\kappa\|_{1, e}^{2}\right)^{1 / 2}\|\boldsymbol{u}\|_{2}\|\| \mathcal{K}_{h} \boldsymbol{u}-\boldsymbol{u}_{h}\|\| .
\end{aligned}
$$

Putting (3.36), (3.35) and (3.34) in (3.33) we find

$$
\begin{aligned}
& -\ell\left(\boldsymbol{w}_{h}\right)+b_{h}\left(\boldsymbol{w}_{h}, p\right)+a_{h}\left(\boldsymbol{u}, \boldsymbol{w}_{h}\right) \\
\leq & \boldsymbol{c h}\left(\|\boldsymbol{u}\|_{2}+\|p\|_{1}+\|\boldsymbol{u}\|_{2}\left(\sum_{e \in \mathcal{E}_{h}^{s}}\|\kappa\|_{1, e}^{2}\right)^{1 / 2}\right)\left\|\mathcal{K}_{h} \boldsymbol{u}-\boldsymbol{u}_{h}\right\| .
\end{aligned}
$$


Returning to (3.25) with (3.26), (3.30), (3.37) and using Cauchy-Schwarz and Young's inequalities we find

$$
\begin{aligned}
\|\| \boldsymbol{u}_{h}-\mathcal{K}_{h} \boldsymbol{u}\|\|^{2} \leq & \boldsymbol{c}\left\|\mathcal{K}_{h} \boldsymbol{u}-\boldsymbol{u}\right\| \|^{2}+\boldsymbol{c}\left(\sum_{e \in \mathcal{E}_{h}^{s}}\left(1+h_{e}^{2}\right)\|g\|_{1, e}^{2}\right)^{1 / 2}\left(\sum_{e \in \mathcal{E}_{h}^{s}}\left\|\left(\mathcal{K}_{h} \boldsymbol{u}\right)_{\boldsymbol{\tau}}-\boldsymbol{u}_{\boldsymbol{\tau}}\right\|_{e}^{2}\right)^{1 / 2} \\
& +\boldsymbol{c h} h^{2}\left(\|\boldsymbol{u}\|_{2}^{2}+\|p\|_{1}^{2}+\sum_{e \in \mathcal{E}_{h}}\|\kappa\|_{1, e}^{2}\right) \\
& +\boldsymbol{c} h\left[h\left(\sum_{e \in \mathcal{E}_{h}^{s}}\|g\|_{1, e}^{2}\right)^{1 / 2}+\left(\sum_{e \in \mathcal{E}_{h}^{s}}\left(h_{e}^{2}+1\right)\|g\|_{1, e}^{2}\right)^{1 / 2}\right]^{2}
\end{aligned}
$$

The error estimate announced is therefore obtained from (3.38) after application of the triangle inequality.

\subsection{A priori error on the pressure}

To estimate the error on the pressure, we proceed as in [10] (see Th. 6, pp 66). We note that $\Pi_{K}^{0} a$ is the mean over $K$ while $\Pi_{e}^{0} a$ is the mean over $e$.

We claim that

Proposition 3.3 Let $(\boldsymbol{f}, g) \in \boldsymbol{L}^{2}(\Omega) \times L^{\infty}(S) \cap H^{1}(S)$. Let $(\boldsymbol{u}, p)$ and $\left(\boldsymbol{u}_{h}, p_{h}\right)$ be solutions of (2.17) and (3.1) respectively. If $(\boldsymbol{u}, p)$ belong to $\boldsymbol{H}^{2}(\Omega) \times H^{1}(\Omega)$, then

$$
\begin{aligned}
\left\|p-p_{h}\right\| \leq & \boldsymbol{c}\left(\sum_{K \in \mathcal{T}}\left\|p-\Pi_{K}^{0} p\right\|_{L^{2}(K)}^{2}\right)^{1 / 2}+\boldsymbol{c}\left\|\boldsymbol{u}-\boldsymbol{u}_{h}\right\| \|+\nu\left(\sum_{e \in \mathcal{E}_{h}} h_{e}\left\|\nabla \boldsymbol{u}-\Pi_{e}^{0} \nabla \boldsymbol{u}\right\|_{e}^{2}\right)^{1 / 2} \\
& +\left(\sum_{e \in \mathcal{E}_{h}} h_{e}\left\|p-\Pi_{e}^{0} p\right\|_{e}^{2}\right)^{1 / 2}
\end{aligned}
$$

where $\boldsymbol{c} \equiv \boldsymbol{c}(\Omega)$ is a positive constant independent of $h$.

proof. We recall $\left(\boldsymbol{u}_{h}, p_{h}\right)$ is defined via (3.1), that is

$$
\left\{\begin{array}{l}
a_{h}\left(\boldsymbol{u}_{h}, \boldsymbol{v}_{h}-\boldsymbol{u}_{h}\right)+b_{h}\left(\boldsymbol{v}_{h}-\boldsymbol{u}_{h}, p_{h}\right)+j_{h}\left(\boldsymbol{v}_{h}\right)-j_{h}\left(\boldsymbol{u}_{h}\right) \geq \ell_{h}\left(\boldsymbol{v}_{h}-\boldsymbol{u}_{h}\right), \\
b_{h}\left(\boldsymbol{u}_{h}, q_{h}\right)=0, \\
\text { for all }\left(q_{h}, \boldsymbol{v}_{h}\right) \in M_{h} \times \boldsymbol{V}_{h} .
\end{array}\right.
$$

We take $\boldsymbol{v}_{h}-\boldsymbol{u}_{h}= \pm \boldsymbol{w}_{h}$ with $\left.\boldsymbol{w}_{h}\right|_{S}=0$ in the first equation of (3.39). This gives

$$
a_{h}\left(\boldsymbol{u}_{h}, \boldsymbol{w}_{h}\right)+b_{h}\left(\boldsymbol{w}_{h}, p_{h}\right)=\ell_{h}\left(\boldsymbol{w}_{h}\right),
$$

which is rewritten thanks to the linearity,

$$
\begin{aligned}
b_{h}\left(\boldsymbol{w}_{h}, p_{h}\right) & =-a_{h}\left(\boldsymbol{u}_{h}, \boldsymbol{w}_{h}\right)+\ell_{h}\left(\boldsymbol{w}_{h}\right) \\
& =-a_{h}\left(\boldsymbol{u}, \boldsymbol{w}_{h}\right)+\ell_{h}\left(\boldsymbol{w}_{h}\right)+a_{h}\left(\boldsymbol{u}-\boldsymbol{u}_{h}, \boldsymbol{w}_{h}\right) .
\end{aligned}
$$


Thus we need to treat $-a_{h}\left(\boldsymbol{u}, \boldsymbol{w}_{h}\right)+\ell_{h}\left(\boldsymbol{w}_{h}\right)$. For that purpose, we recall that since $(\boldsymbol{u}, p) \in \boldsymbol{H}^{2} \times H^{1}$, $[\nabla \boldsymbol{u}]=\mathbf{0},[p]=\mathbf{0},\{\nabla \boldsymbol{u}\}=\nabla \boldsymbol{u}$ and $\{p\}=p$. Hence using (2.57) and (2.11)

$$
\begin{aligned}
-a_{h}\left(\boldsymbol{u}, \boldsymbol{w}_{h}\right)+\ell_{h}\left(\boldsymbol{w}_{h}\right) & =-\nu \sum_{K} \int_{K} \nabla \boldsymbol{u}: \nabla \boldsymbol{w}_{h}+\sum_{K} \int_{K} \boldsymbol{f} \cdot \boldsymbol{w}_{h} \\
& =\nu \sum_{K} \int_{K} \Delta \boldsymbol{u} \cdot \boldsymbol{w}_{h}-\nu \sum_{e \in \mathcal{E}_{h}} \int_{e}\{\nabla \boldsymbol{u}\}:\left[\underline{\boldsymbol{w}_{h}}\right]-\nu \sum_{e \in \mathcal{E}_{h}^{0}} \int_{e}[\nabla \boldsymbol{u}] \cdot\left\{\boldsymbol{w}_{h}\right\}+\sum_{K} \int_{K} \boldsymbol{f} \cdot \boldsymbol{w}_{h} \\
& =\nu \sum_{K} \int_{K} \Delta \boldsymbol{u} \cdot \boldsymbol{w}_{h}-\nu \sum_{e \in \mathcal{E}_{h}} \int_{e} \nabla \boldsymbol{u}:\left[\underline{\boldsymbol{w}_{h}}\right]+\sum_{K} \int_{K} \boldsymbol{f} \cdot \boldsymbol{w}_{h} \\
& =\sum_{K} \int_{K} \nabla p \cdot \boldsymbol{w}_{h}-\nu \sum_{e \in \mathcal{E}_{h}} \int_{e} \nabla \boldsymbol{u}:\left[\underline{\boldsymbol{w}_{h}}\right] \\
& =-\sum_{K} \int_{K} p \operatorname{div} \boldsymbol{w}_{h}+\sum_{e \in \mathcal{E}_{h}} \int_{e}\{p\}\left[\boldsymbol{w}_{h}\right]+\sum_{e \in \mathcal{E}_{h}^{0}} \int_{e}[p] \cdot\left\{\boldsymbol{w}_{h}\right\}-\nu \sum_{e \in \mathcal{E}_{h}} \int_{e} \nabla \boldsymbol{u}:\left[\underline{\boldsymbol{w}_{h}}\right] \\
& =b_{h}\left(\boldsymbol{w}_{h}, p\right)-\sum_{e \in \mathcal{E}_{h}} \int_{e}(\nu \nabla \boldsymbol{u}-p \boldsymbol{I}):\left[\underline{\left.\boldsymbol{w}_{h}\right]} .\right.
\end{aligned}
$$

Inserting (3.41) in (3.40), and using (2.54) gives

$$
\begin{aligned}
b_{h}\left(\boldsymbol{w}_{h}, p_{h}\right)= & b_{h}\left(\boldsymbol{w}_{h}, p\right)-\sum_{e \in \mathcal{E}_{h}} \int_{e}(\nu \nabla \boldsymbol{u}-p \boldsymbol{I}):\left[\underline{\boldsymbol{w}_{h}}\right]+a_{h}\left(\boldsymbol{u}-\boldsymbol{u}_{h}, \boldsymbol{w}_{h}\right) \\
= & \left.b_{h}\left(\boldsymbol{w}_{h}, p-\Pi_{h} p\right)+b_{h}\left(\boldsymbol{w}_{h}, \Pi_{h} p\right)-\sum_{e \in \mathcal{E}_{h}} \int_{e}(\nu \nabla \boldsymbol{u}-p \boldsymbol{I}): \underline{\underline{\boldsymbol{w}_{h}}}\right]+a_{h}\left(\boldsymbol{u}-\boldsymbol{u}_{h}, \boldsymbol{w}_{h}\right) \\
= & b_{h}\left(\boldsymbol{w}_{h}, p-\Pi_{h} p\right)+b_{h}\left(\boldsymbol{w}_{h}, \Pi_{h} p\right)-\nu \sum_{e \in \mathcal{E}_{h}} \int_{e}\left(\nabla \boldsymbol{u}-\Pi_{e}^{0} \nabla \boldsymbol{u}\right):\left[\underline{\boldsymbol{w}_{h}}\right]+\sum_{e \in \mathcal{E}_{h}} \int_{e}\left(p-\Pi^{0} p\right) \boldsymbol{I}:\left[\underline{\boldsymbol{w}_{h}}\right] \\
& +a_{h}\left(\boldsymbol{u}-\boldsymbol{u}_{h}, \boldsymbol{w}_{h}\right),
\end{aligned}
$$

which is re-written as follows

$$
\begin{aligned}
b_{h}\left(\boldsymbol{w}_{h}, p_{h}-\Pi_{h} p\right)= & b_{h}\left(\boldsymbol{w}_{h}, p-\Pi_{h} p\right)-\nu \sum_{e \in \mathcal{E}_{h}} \int_{e}\left(\nabla \boldsymbol{u}-\Pi_{e}^{0} \nabla \boldsymbol{u}\right):\left[\underline{\boldsymbol{w}_{h}}\right]+\sum_{e \in \mathcal{E}_{h}} \int_{e}\left(p-\Pi^{0} p\right) \boldsymbol{I}:\left[\underline{\boldsymbol{w}_{h}}\right] \\
& +a_{h}\left(\boldsymbol{u}-\boldsymbol{u}_{h}, \boldsymbol{w}_{h}\right) .
\end{aligned}
$$

Next, we recall that since $\left(p_{h}-\Pi_{h} p, 1\right)=0$, and $\left(\boldsymbol{V}_{h}, M_{h}\right)$ is inf-sup stable, one can find $\boldsymbol{w}_{h} \in$ $\boldsymbol{V}_{h} \cap\left\{\left.\boldsymbol{w}_{h}\right|_{S}=0\right\}$ such that

$$
\left\{\begin{array}{l}
\operatorname{div}_{h} \boldsymbol{w}_{h}=p_{h}-\Pi_{K}^{h} p \text { on } K, \\
\left\|\mid \boldsymbol{w}_{h}\right\| \leq \boldsymbol{c}\left\|p_{h}-\Pi_{h} p\right\| .
\end{array}\right.
$$


Putting together (3.42) and (3.43), one finds

$$
\begin{aligned}
\left\|p_{h}-\Pi_{h} p\right\|^{2}= & \sum_{K}\left\|p_{h}-\Pi_{K}^{h} p\right\|_{L^{2}(K)}^{2} \\
= & \sum_{K \in \mathcal{T}} \int_{K}\left(p_{h}-\Pi_{K}^{h} p\right) \operatorname{div} \boldsymbol{w}_{h} \\
= & -b_{h}\left(\boldsymbol{w}_{h}, p_{h}-\Pi_{h} p\right) \\
= & -b_{h}\left(\boldsymbol{w}_{h}, p-\Pi_{h}\right)-a_{h}\left(\boldsymbol{u}-\boldsymbol{u}_{h}, \boldsymbol{w}_{h}\right)+\nu \sum_{e \in \mathcal{E}_{h}} \int_{e}\left(\nabla \boldsymbol{u}-\Pi_{e}^{0} \nabla \boldsymbol{u}\right):\left[\underline{\boldsymbol{w}_{h}}\right] \\
& -\sum_{e \in \mathcal{E}_{h}} \int_{e}\left(p-\Pi_{e}^{0} p\right) \boldsymbol{I}:\left[\underline{\boldsymbol{w}_{h}}\right] \\
\leq & \boldsymbol{c}\left\|\boldsymbol{w}_{h}\right\|\|\| p-\Pi_{h} p\|+\boldsymbol{c}\|\left\|\boldsymbol{u}-\boldsymbol{u}_{h}\right\|\|\| \mid \boldsymbol{w}_{h}\|\|+\nu\left[\sum_{e \in \mathcal{E}_{h}} h_{e}\left\|\nabla \boldsymbol{u}-\Pi_{e}^{0} \nabla \boldsymbol{u}\right\|_{e}^{2}\right]^{1 / 2}\left[\sum_{e \in \mathcal{E}_{h}} \frac{1}{h_{e}}\left\|\left[\underline{\boldsymbol{w}}_{h}\right]\right\|_{e}^{2}\right]^{1 / 2} \\
& +\left[\sum_{e \in \mathcal{E}_{h}} h_{e}\left\|p-\Pi_{e}^{0} p\right\|_{e}^{2}\right]^{1 / 2}\left[\sum_{e \in \mathcal{E}_{h}} \frac{1}{h_{e}}\left\|\left[\underline{\boldsymbol{w}}_{h}\right]\right\|_{e}^{2}\right]^{1 / 2} \\
\leq & \boldsymbol{c}\left\|p_{h}-\Pi_{h} p\right\|\left\|p-\Pi_{h} p\right\|+C\left\|\boldsymbol{u}-\boldsymbol{u}_{h}\right\|\|\| p_{h}-\Pi_{h} p \| \\
& +\nu\left[\sum_{e \in \mathcal{E}_{h}} h_{e}\left\|\nabla \boldsymbol{u}-\Pi_{e}^{0} \nabla \boldsymbol{u}\right\|_{e}^{2}\right]^{1 / 2}\left\|p_{h}-\Pi_{h} p\right\|+\left[\sum_{e \in \mathcal{E}_{h}} h_{e}\left\|p-\Pi_{e}^{0} p\right\|_{e}^{2}\right]^{1 / 2}\left\|p_{h}-\Pi_{h} p\right\|
\end{aligned}
$$

That is

$$
\begin{aligned}
\left\|p_{h}-\Pi_{h} p\right\| \leq & c\left\|p-\Pi_{h} p\right\|+\boldsymbol{c}\left\|\boldsymbol{u}-\boldsymbol{u}_{h} \mid\right\|+\nu\left[\sum_{e \in \mathcal{E}_{h}} h_{e}\left\|\nabla \boldsymbol{u}-\Pi_{e}^{0} \nabla \boldsymbol{u}\right\|_{e}^{2}\right]^{1 / 2} \\
& +\left[\sum_{e \in \mathcal{E}_{h}} h_{e}\left\|p-\Pi_{e}^{0} p\right\|_{e}^{2}\right]^{1 / 2}
\end{aligned}
$$

So, the desired result is obtained by combining (3.44) and the triangle's inequality.

\section{Navier-Stokes system: A Priori Error Estimate}

\subsection{Some preliminaries}

We define the trilinear form $d_{h}(\cdot, \cdot, \cdot)$ as

$$
d_{h}(\boldsymbol{u}, \boldsymbol{v}, \boldsymbol{w})=\sum_{K} \int_{K}(\boldsymbol{u} \cdot \nabla) \boldsymbol{v} \cdot \boldsymbol{w} d x
$$

The finite element solution associated to Problem (F) is defined as follows

$$
\left\{\begin{array}{l}
\operatorname{Problem}(\mathbf{F h}) \\
\text { Find }\left(\boldsymbol{u}_{h}, p_{h}\right) \in \boldsymbol{V}_{h} \times M_{h} \text { that satisfies, } \\
a_{h}\left(\boldsymbol{u}_{h}, \boldsymbol{v}_{h}-\boldsymbol{u}_{h}\right)+d_{h}\left(\boldsymbol{u}_{h}, \boldsymbol{u}_{h}, \boldsymbol{v}_{h}-\boldsymbol{u}_{h}\right)+b_{h}\left(\boldsymbol{v}_{h}-\boldsymbol{u}_{h}, p_{h}\right) \\
+j_{h}\left(\boldsymbol{v}_{h}\right)-j_{h}\left(\boldsymbol{u}_{h}\right) \geq \ell_{h}\left(\boldsymbol{v}_{h}-\boldsymbol{u}_{h}\right), \\
b_{h}\left(\boldsymbol{u}_{h}, q_{h}\right)=0, \\
\text { for all }\left(\boldsymbol{v}_{h}, q_{h}\right) \in \boldsymbol{V}_{h} \times M_{h} .
\end{array}\right.
$$


The solvability of (4.1) is obtained by following the same steps as in the proof of Proposition 2.2, and the analogue of Proposition 2.2 can be stated as follows

Proposition 4.1 Let $(\boldsymbol{f}, g) \in \boldsymbol{L}^{2}(\Omega) \times L^{\infty}(S)$. Then problem (4.1) has at least one solution $\left(\boldsymbol{u}_{h}, p_{h}\right) \in \boldsymbol{V}_{h} \times M_{h}$, and the following a priori estimates hold

$$
\left\|\boldsymbol{u}_{h}\right\| \mid \leq \frac{\boldsymbol{c}}{\nu}\|\boldsymbol{f}\|, \quad\left\|p_{h}\right\| \leq\left(1+\kappa_{0}\right) \boldsymbol{c}\|\boldsymbol{f}\|+\frac{\boldsymbol{c}}{\nu^{2}}\|\boldsymbol{f}\|^{2} .
$$

If moreover the viscosity $\nu$ is taken in such a way that (2.28) holds, then the solution is unique.

The proof of (4.2) is done as in the continuous case, hence will not be repeated here.

\subsection{A priori error on the velocity}

The main result of the paragraph can be stated as follows

Proposition 4.2 Let $(\boldsymbol{f}, g) \in \boldsymbol{L}^{2}(\Omega) \times L^{\infty}(S) \cap H^{1}(S)$. Assume that (2.28) holds. Let $(\boldsymbol{u}, p)$ be the solution of (2.27). Let $\left(\boldsymbol{u}_{h}, p_{h}\right)$ be solution of (3.1). If $(\boldsymbol{u}, p)$ belong to $\boldsymbol{H}^{2}(\Omega) \times H^{1}(\Omega)$, then

$$
\begin{aligned}
\|\| \boldsymbol{u}_{h}-\boldsymbol{u} \| \mid \leq & \boldsymbol{c}\left[\sum_{e \in \mathcal{E}_{h}^{s}}\left(1+h_{e}^{2}\right)\|g\|_{1, e}^{2}\right]^{1 / 4}\left[\sum_{e \in \mathcal{E}_{h}^{s}}\left\|\left(\mathcal{K}_{h} \boldsymbol{u}\right)_{\boldsymbol{\tau}}-\boldsymbol{u}_{\boldsymbol{\tau}}\right\|_{e}^{2}\right]^{1 / 4}+\boldsymbol{c}\left\|\boldsymbol{u}-\mathcal{K}_{h} \boldsymbol{u}\right\| \| \\
& +\boldsymbol{c} h^{1 / 2}\left[h\left(\sum_{e \in \mathcal{E}_{h}^{s}}\|g\|_{1, e}^{2}\right)^{1 / 2}+\left(\sum_{e \in \mathcal{E}_{h}^{s}}\left(h_{e}^{2}+1\right)\|g\|_{1, e}^{2}\right)^{1 / 2}\right] \\
& +\boldsymbol{c h}\left(\|\boldsymbol{u}\|_{2}+\|p\|_{1}+\|\boldsymbol{u}\|_{2}\left(\sum_{e \in \mathcal{E}_{h}^{s}}\|\kappa\|_{1, e}^{2}\right)^{1 / 2}\right),
\end{aligned}
$$

where $\boldsymbol{c}$ is a positive constant independent of $h$.

Proof. We proceed as in the proof of Proposition 3.2

The first step in the proof is unchanged. Hence (3.18) is valid here.

Step 2: Use of coercivity condition on $a_{h}(\cdot, \cdot)$.

From the coercivity of $a_{h}(\cdot, \cdot)$ and the first equation in (4.1), and (3.20), and (3.22) we obtain

$$
\begin{aligned}
\nu\left\|\boldsymbol{u}_{h}-\mathcal{K}_{h} \boldsymbol{u}\right\| \|^{2} \leq & a_{h}\left(\boldsymbol{u}_{h}-\mathcal{K}_{h} \boldsymbol{u}, \boldsymbol{u}_{h}-\mathcal{K}_{h} \boldsymbol{u}\right) \\
= & a_{h}\left(\boldsymbol{u}_{h}, \boldsymbol{u}_{h}-\mathcal{K}_{h} \boldsymbol{u}\right)-a_{h}\left(\boldsymbol{v}_{h}, \boldsymbol{u}_{h}-\mathcal{K}_{h} \boldsymbol{u}\right) \\
\leq & d_{h}\left(\boldsymbol{u}_{h}, \boldsymbol{u}_{h}, \mathcal{K}_{h} \boldsymbol{u}-\boldsymbol{u}_{h}\right)+j_{h}\left(\mathcal{K}_{h} \boldsymbol{u}\right)-j_{h}\left(\boldsymbol{u}_{h}\right)-\ell_{h}\left(\mathcal{K}_{h} \boldsymbol{u}-\boldsymbol{u}_{h}\right)-a_{h}\left(\mathcal{K}_{h} \boldsymbol{u}, \boldsymbol{u}_{h}-\mathcal{K}_{h} \boldsymbol{u}\right) \\
= & d_{h}\left(\boldsymbol{u}_{h}, \boldsymbol{u}_{h}, \mathcal{K}_{h} \boldsymbol{u}-\boldsymbol{u}_{h}\right)-\ell\left(\mathcal{K}_{h} \boldsymbol{u}-\boldsymbol{u}_{h}-E_{h}\left(\mathcal{K}_{h} \boldsymbol{u}-\boldsymbol{u}_{h}\right)\right)-a_{h}\left(\mathcal{K}_{h} \boldsymbol{u}-\boldsymbol{u}, \boldsymbol{u}_{h}-\mathcal{K}_{h} \boldsymbol{u}\right) \\
& -a_{h}\left(\boldsymbol{u}, \boldsymbol{u}_{h}-\mathcal{K}_{h} \boldsymbol{u}\right)+j_{h}\left(\mathcal{K}_{h} \boldsymbol{u}\right)-j_{h}\left(\boldsymbol{u}_{h}\right)-\ell\left(E_{h}\left(\mathcal{K}_{h} \boldsymbol{u}-\boldsymbol{u}_{h}\right)\right) \\
= & -\ell\left(\mathcal{K}_{h} \boldsymbol{u}-\boldsymbol{u}_{h}-E_{h}\left(\mathcal{K}_{h} \boldsymbol{u}-\boldsymbol{u}_{h}\right)\right)+b_{h}\left(\mathcal{K}_{h} \boldsymbol{u}-\boldsymbol{u}_{h}, p\right)+d_{h}\left(\boldsymbol{u}_{h}, \boldsymbol{u}_{h}, \mathcal{K}_{h} \boldsymbol{u}-\boldsymbol{u}_{h}\right) \\
& -a_{h}\left(\boldsymbol{u}, \boldsymbol{u}_{h}-\mathcal{K}_{h} \boldsymbol{u}\right)+j_{h}\left(\mathcal{K}_{h} \boldsymbol{u}\right)-j_{h}\left(\boldsymbol{u}_{h}\right)-\ell\left(E_{h}\left(\mathcal{K}_{h} \boldsymbol{u}-\boldsymbol{u}_{h}\right)\right) .
\end{aligned}
$$


Now in the first equation of (2.47), letting $\boldsymbol{v}=E_{h}\left(\mathcal{K}_{h} \boldsymbol{u}-\boldsymbol{u}_{h}\right) \in \boldsymbol{V}_{h}^{c} \subset \boldsymbol{V}$, we obtain that

$$
\begin{aligned}
\ell\left(E_{h}\left(\mathcal{K}_{h} \boldsymbol{u}-\boldsymbol{u}_{h}\right)\right)= & a\left(\boldsymbol{u}, E_{h}\left(\mathcal{K}_{h} \boldsymbol{u}-\boldsymbol{u}_{h}\right)\right)+d\left(\boldsymbol{u}, \boldsymbol{u}, E_{h}\left(\mathcal{K}_{h} \boldsymbol{u}-\boldsymbol{u}_{h}\right)\right)+b\left(E_{h}\left(\mathcal{K}_{h} \boldsymbol{u}-\boldsymbol{u}_{h}\right), p\right) \\
& +\left(g \boldsymbol{\alpha}, E_{\boldsymbol{\tau}, h}\left(\mathcal{K}_{h} \boldsymbol{u}-\boldsymbol{u}_{h}\right)\right)_{S}
\end{aligned}
$$

Inserting (4.4) in (4.3), one obtains

$$
\begin{aligned}
& \nu\left\|\boldsymbol{u}_{h}-\mathcal{K}_{h} \boldsymbol{u}\right\| \|^{2} \\
\leq \quad & -\ell\left(\mathcal{K}_{h} \boldsymbol{u}-\boldsymbol{u}_{h}-E_{h}\left(\mathcal{K}_{h} \boldsymbol{u}-\boldsymbol{u}_{h}\right)\right)+b_{h}\left(\mathcal{K}_{h} \boldsymbol{u}-\boldsymbol{u}_{h}-E_{h}\left(\mathcal{K}_{h} \boldsymbol{u}-\boldsymbol{u}_{h}\right), p\right)+d\left(\boldsymbol{u}, \boldsymbol{u}, \mathcal{K}_{h} \boldsymbol{u}-\boldsymbol{u}_{h}-E_{h}\left(\mathcal{K}_{h} \boldsymbol{u}-\boldsymbol{u}_{h}\right)\right) \\
& +a_{h}\left(\boldsymbol{u}, \mathcal{K}_{h} \boldsymbol{u}-\boldsymbol{u}_{h}-E_{h}\left(\mathcal{K}_{h} \boldsymbol{u}-\boldsymbol{u}_{h}\right)\right)-\left(g \boldsymbol{\alpha}, E_{\boldsymbol{\tau}, h}\left(\mathcal{K}_{h} \boldsymbol{u}-\boldsymbol{u}_{h}\right)-\left(\mathcal{K}_{h} \boldsymbol{u}-\boldsymbol{u}_{h}\right)_{\boldsymbol{\tau}}\right)_{S} \\
& +d\left(\boldsymbol{u}, \boldsymbol{u}, \boldsymbol{u}_{h}-\mathcal{K}_{h} \boldsymbol{u}\right)-d_{h}\left(\boldsymbol{u}_{h}, \boldsymbol{u}_{h}, \boldsymbol{u}_{h}-\mathcal{K}_{h} \boldsymbol{u}\right)+j_{h}\left(\mathcal{K}_{h} \boldsymbol{u}\right)-j_{h}\left(\boldsymbol{u}_{h}\right)-\left(g \boldsymbol{\alpha},\left(\mathcal{K}_{h} \boldsymbol{u}-\boldsymbol{u}_{h}\right)_{\boldsymbol{\tau}}\right)_{S} .
\end{aligned}
$$

We now want to estimate terms on the right hand side of (4.5). First, we have established that (see $(3.26),(3.30))$

$$
\begin{aligned}
& j_{h}\left(\mathcal{K}_{h} \boldsymbol{u}\right)-j_{h}\left(\boldsymbol{u}_{h}\right)-\left(g \boldsymbol{\alpha},\left(\mathcal{K}_{h} \boldsymbol{u}-\boldsymbol{u}_{h}\right)_{\boldsymbol{\tau}}\right)_{S} \\
\leq & \boldsymbol{c}\left[\sum_{e \in \mathcal{E}_{h}^{s}}\left(1+h_{e}^{2}\right)\|g\|_{1, e}^{2}\right]^{1 / 2}\left[\sum_{e \in \mathcal{E}_{h}^{s}}\left\|\left(\mathcal{K}_{h} \boldsymbol{u}\right)_{\boldsymbol{\tau}}-\boldsymbol{u}_{\boldsymbol{\tau}}\right\|_{e}^{2}\right]^{1 / 2}, \\
& \text { and } \\
& -\left(g \boldsymbol{\alpha}, E_{\boldsymbol{\tau}, h}\left(\mathcal{K}_{h} \boldsymbol{u}-\boldsymbol{u}_{h}\right)-\left(\mathcal{K}_{h} \boldsymbol{u}-\boldsymbol{u}_{h}\right)_{\boldsymbol{\tau}}\right)_{S} \\
\leq & \boldsymbol{c h}^{1 / 2}\left[h\left(\sum_{e \in \mathcal{E}_{h}^{s}}\|g\|_{1, e}^{2}\right)^{1 / 2}+\left(\sum_{e \in \mathcal{E}_{h}^{s}}\left(h_{e}^{2}+1\right)\|g\|_{1, e}^{2}\right)^{1 / 2}\right]\left\|\mathcal{K}_{h} \boldsymbol{u}-\boldsymbol{u}_{h}\right\| \| .
\end{aligned}
$$

From the properties of $d(\cdot, \cdot)$ together with $(2.1)$ and $(4.2)$, one has

$$
\begin{aligned}
& d\left(\boldsymbol{u}, \boldsymbol{u}, \boldsymbol{u}_{h}-\mathcal{K}_{h} \boldsymbol{u}\right)-d_{h}\left(\boldsymbol{u}_{h}, \boldsymbol{u}_{h}, \boldsymbol{u}_{h}-\mathcal{K}_{h} \boldsymbol{u}\right) \\
= & d\left(\boldsymbol{u}, \boldsymbol{u}-\mathcal{K}_{h} \boldsymbol{u}, \boldsymbol{u}_{h}-\mathcal{K}_{h} \boldsymbol{u}\right)+d\left(\boldsymbol{u}-\mathcal{K}_{h} \boldsymbol{u}, \boldsymbol{u}_{h}, \boldsymbol{u}_{h}-\mathcal{K}_{h} \boldsymbol{u}\right)+d\left(\mathcal{K}_{h} \boldsymbol{u}-\boldsymbol{u}_{h}, \boldsymbol{u}_{h}, \boldsymbol{u}_{h}-\mathcal{K}_{h} \boldsymbol{u}\right) \\
\leq & \boldsymbol{c}\|\nabla \boldsymbol{u}\|\left\|\boldsymbol{u}-\mathcal{K}_{h} \boldsymbol{u}\right\|\|\| \boldsymbol{u}_{h}-\mathcal{K}_{h} \boldsymbol{u}\|\mid+\boldsymbol{c}\| \boldsymbol{u}-\mathcal{K}_{h} \boldsymbol{u}\|\|\left\|\boldsymbol{u}_{h}-\mathcal{K}_{h} \boldsymbol{u}\right\|\|\| \nabla \boldsymbol{u}_{h} \| \\
& +\left.\boldsymbol{c}\left\|\mathcal{K}_{h} \boldsymbol{u}-\boldsymbol{u}_{h}\right\|\right|^{2}\left\|\nabla \boldsymbol{u}_{h}\right\| \\
\leq & \frac{\boldsymbol{c}}{\nu}\|\boldsymbol{f}\|\|\| \boldsymbol{u}-\mathcal{K}_{h} \boldsymbol{u}\|\|\left\|\boldsymbol{u}_{h}-\mathcal{K}_{h} \boldsymbol{u}\right\|+\frac{\boldsymbol{c}}{\nu}\|\boldsymbol{f}\|\left\|\mathcal{K}_{h} \boldsymbol{u}-\boldsymbol{u}_{h}\right\| \|^{2}
\end{aligned}
$$

Thirdly knowing that $(\boldsymbol{u}, p) \in \boldsymbol{H}^{2} \times H^{1}$ and following the way we have derived (3.37), we deduce that

$$
\begin{aligned}
& -\ell\left(\mathcal{K}_{h} \boldsymbol{u}-\boldsymbol{u}_{h}-E_{h}\left(\mathcal{K}_{h} \boldsymbol{u}-\boldsymbol{u}_{h}\right)\right)+b_{h}\left(\mathcal{K}_{h} \boldsymbol{u}-\boldsymbol{u}_{h}-E_{h}\left(\mathcal{K}_{h} \boldsymbol{u}-\boldsymbol{u}_{h}\right), p\right)+d\left(\boldsymbol{u}, \boldsymbol{u}, \mathcal{K}_{h} \boldsymbol{u}-\boldsymbol{u}_{h}-E_{h}\left(\mathcal{K}_{h} \boldsymbol{u}-\boldsymbol{u}_{h}\right)\right) \\
& +a_{h}\left(\boldsymbol{u}, \mathcal{K}_{h} \boldsymbol{u}-\boldsymbol{u}_{h}-E_{h}\left(\mathcal{K}_{h} \boldsymbol{u}-\boldsymbol{u}_{h}\right)\right) \\
= & \sum_{e \in \mathcal{E}_{h}} \int_{e}\left[\mathcal{K}_{h} \boldsymbol{u}-\boldsymbol{u}_{h}-E_{h}\left(\mathcal{K}_{h} \boldsymbol{u}-\boldsymbol{u}_{h}\right)\right]:(\nu \nabla \boldsymbol{u}-p \boldsymbol{I}) d x+\nu \sum_{e} \int_{e} \kappa\left(\mathcal{K}_{h} \boldsymbol{u}-\boldsymbol{u}_{h}-E_{h}\left(\mathcal{K}_{h} \boldsymbol{u}-\boldsymbol{u}_{h}\right)\right)_{\boldsymbol{\tau}} \cdot \boldsymbol{u}_{\boldsymbol{\tau}} \\
\leq & \boldsymbol{c h}\left(\|\boldsymbol{u}\|_{2}+\|p\|_{1}+\|\boldsymbol{u}\|_{2}\left(\sum_{e \in \mathcal{E}_{h}^{s}}\|\kappa\|_{1, e}^{2}\right)^{1 / 2}\right)\|\| \mathcal{K}_{h} \boldsymbol{u}-\boldsymbol{u}_{h}\|\| .
\end{aligned}
$$


Returning to (4.5) with (4.8), (4.7), (4.6) we find

$$
\begin{aligned}
& \left(\nu-\frac{\boldsymbol{c}}{\nu}\|\boldsymbol{f}\|\right)\|\| \boldsymbol{u}_{h}-\mathcal{K}_{h} \boldsymbol{u} \|^{2} \\
\leq & \boldsymbol{c}\left[\sum_{e \in \mathcal{E}_{h}^{s}}\left(1+h_{e}^{2}\right)\|g\|_{1, e}^{2}\right]^{1 / 2}\left[\sum_{e \in \mathcal{E}_{h}^{s}}\left\|\left(\mathcal{K}_{h} \boldsymbol{u}\right)_{\boldsymbol{\tau}}-\boldsymbol{u}_{\boldsymbol{\tau}}\right\|_{e}^{2}\right]^{1 / 2}+\frac{\boldsymbol{c}}{\nu}\|\boldsymbol{f}\|\|\| \boldsymbol{u}-\mathcal{K}_{h} \boldsymbol{u}\|\|\left\|\boldsymbol{u}_{h}-\mathcal{K}_{h} \boldsymbol{u}\right\| \\
& +\boldsymbol{c} h^{1 / 2}\left[h\left(\sum_{e \in \mathcal{E}_{h}^{s}}\|g\|_{1, e}^{2}\right)^{1 / 2}+\left(\sum_{e \in \mathcal{E}_{h}^{s}}\left(h_{e}^{2}+1\right)\|g\|_{1, e}^{2}\right)^{1 / 2}\right]\left\|\mathcal{K}_{h} \boldsymbol{u}-\boldsymbol{u}_{h}\right\| \| \\
& +\boldsymbol{c h}\left(\|\boldsymbol{u}\|_{2}+\|p\|_{1}+\|\boldsymbol{u}\|_{2}\left(\sum_{e \in \mathcal{E}_{h}^{s}}\|\kappa\|_{1, e}^{2}\right)^{1 / 2}\right)\|\| \mathcal{K}_{h} \boldsymbol{u}-\boldsymbol{u}_{h}\|\| .
\end{aligned}
$$

Clearly, taking into account (2.28), and using Young's inequality, (4.9) gives

$$
\begin{aligned}
\left\|\boldsymbol{u}_{h}-\mathcal{K}_{h} \boldsymbol{u}\right\|^{2} \leq & \boldsymbol{c}\left[\sum_{e \in \mathcal{E}_{h}^{s}}\left(1+h_{e}^{2}\right)\|g\|_{1, e}^{2}\right]^{1 / 2}\left[\sum_{e \in \mathcal{E}_{h}^{s}}\left\|\left(\mathcal{K}_{h} \boldsymbol{u}\right)_{\boldsymbol{\tau}}-\boldsymbol{u}_{\boldsymbol{\tau}}\right\|_{e}^{2}\right]^{1 / 2}+\boldsymbol{c}\left\|\boldsymbol{u}-\mathcal{K}_{h} \boldsymbol{u}\right\|^{2} \\
& +\boldsymbol{c} h\left[h\left(\sum_{e \in \mathcal{E}_{h}^{s}}\|g\|_{1, e}^{2}\right)^{1 / 2}+\left(\sum_{e \in \mathcal{E}_{h}^{s}}\left(h_{e}^{2}+1\right)\|g\|_{1, e}^{2}\right)^{1 / 2}\right]^{2} \\
& +\boldsymbol{c} h^{2}\left(\|\boldsymbol{u}\|_{2}+\|p\|_{1}+\|\boldsymbol{u}\|_{2}\left(\sum_{e \in \mathcal{E}_{h}^{s}}\|\kappa\|_{1, e}^{2}\right)^{1 / 2}\right)^{2}
\end{aligned}
$$

with $c$ a positive constant independent of $h$. We then deduce the result by application of the triangle inequality.

Remark 4.1 the error on the pressure is obtained as in Proposition 4.2.

\section{Conclusion}

In this work, we have established suboptimal a priori estimates for non conforming approximations of the steady incompressible Stokes and Navier-Stokes equations in two dimensions driven by nonlinear slip boundary condition of friction type. We have assumed maximal regularity of the solution and take advantage of the fact that the threshold function $g$ belong to $L^{\infty}(S) \cap H^{1}(S)$, which has allowed us to use sharp estimates. To our knowledge, this is the first analysis of non conforming finite element method using the Crouzeix-Raviart element for the velocity and constant pressure for this type of problems. Our future goals are to study the error estimates with minimal regularity of the solution, efficient solution procedures for their implementation.

\section{References}

[1] H. Fujita. A mathematical analysis of motions of viscous incompressible fluid under leak or slip boundary conditions. In:Mathematical Fluid Mechanics and Modeling, RIMS Kōkyūroko, 
888, Kyoto University, Kyoto, 199-216, 1994.

[2] H. Fujita. Non-stationary Stokes flows under leak boundary conditions of friction type. J. Comput. Appl. Math, 19, 1-8, 2001.

[3] H. Fujita. Variational inequalities and nonlinear semi-groups applied to certain nonlinear problems for the Stokes equation. In: Tosio Kato's Method and Principle for Evolution Equations in Mathematical Physics (Sapporo, 2001), Eds. H. Fujita et al., RIMS, Kyoto, 70-85, 2002.

[4] H. Fujita. A coherent analysis of Stokes flows under boundary conditions of friction type. J. Comput. Appl. Math, 149, 57-69, 2002.

[5] N. Saito. On the Stokes equations with the leak and slip boundary conditions of friction type: regularity of solutions. Publications of RIMS, Kyoto University, 40, 345-383, 2004.

[6] C. Le Roux. Steady Stokes flows with threshold slip boundary conditions. Math. Models Methods Appl. Sci, 15, 1141-1168, 2005.

[7] C. Le Roux, A. Tani. Steady solutions of the Navier-Stokes equations with threshold boundary conditions. Math. Meth. Appl. Sci 30, 595-624, 2007.

[8] V. Girault \& P.A. Raviart. Finite Element Methods for Navier-Stokes Equations: Theory and Algorithms. Springer-Verlag, Berlin, Heidelberg, New-York, Tokyo 1986.

[9] G. Duvaut \& J.-L. Lions. Inequalities in Mechanics and Physics. Grundlehren der Mathematischen Wissenschaften. 219, Springer-Verlag, Berlin, 1976.

[10] M. Crouzeix \& P.A. Raviart. Conforming and nonconforming finite element methods for solving the stationary Stokes equations. R.A.I.R.O. Anal. Numer, 7, 33-76, 1973

[11] J.K. Djoko. Discontinuous Galerkin Finite element discretization for steady Stokes flows with threshold slip boundary conditions. Quaest. Math, 36, 501-516, 2013.

[12] Y. Li \& K. Li. Penalty finite element method for Stokes problem with nonlinear slip boundary conditions. Appl. Math. Comput, 204, 216-226, 2008.

[13] J.K. Djoko \& M. Mbehou. Finite element analysis for Stokes and Navier-Stokes equations driven by threshold slip boundary conditions. IJNAM series B, 4, no 3, 235-255, 2013.

[14] Y. Li \& R. An. Penalty finite element method for Navier-Stokes equations with nonlinear slip boundary conditions. Internat. J. Numer. Methods Fluids, 69, no 3, 550-566, 2012.

[15] Yuan Li \& Katai Li. Semi-discrete finite element methods for Navier-Stokes equations with nonlinear slip boundary conditions based on regularization procedure. Numer. Math, 117, $1-36,2011$.

[16] M. Ayadi, M.K. Gdoura \& T. Sassi. Mixed formulation for Stokes problem with Tresca friction. C.R. Acad. Sci. Paris, Ser. I 348, 1069-1072, 2010.

[17] M. Ayadi, L. Baffico, M. K. Gdoura \& T. Sassi. Error estimates for Stokes problem with tresca friction conditions. Esaim: M2AN 48, 1413-1429, 2014.

[18] T. Kashiwabara. On a finite element approximation of the Stokes equations under a slip boundary condition of the friction type. J. Indust. Appl. Math, 30, 227-261, 2013. 
[19] R. Glowinski. Numerical Methods for Nonlinear Variational Problems. Springer Series in Computational Physics, Springer-Verlag, New York, 1984.

[20] R. Glowinski \& J.-L. Lions \& R.Trémolières. Numerical Analysis of Variational Inequalities, Studies in Mathematics and its Applications, 8, North-Holland, Amsterdam, 1981.

[21] M. Sofonea, \& W. Han \& M. Shillor. Analysis and approximation of contact problems with adhesion or damage, Pure and Applied Mathematics, 276, Chapman \& Hall/CRC, Boca Raton, FL, 2006.

[22] H. Fujita \& H. Kawarada \& A. Sasamoto. Analytical and numerical approaches to stationary flow problems with leak and slip boundary conditions. In: Advances in Numerical Mathematics (Tokyo, 1994), Lect. Notes Numer. Appl. Anal., 14, Eds. T. Ushijima et al., Kinokuniya, 17$31,1995$.

[23] B. D. Reddy. Mixed variational inequalities arising in elastoplasticity. Nonlinear Anal, 19, No 11, 1071-1089, 1992.

[24] W. Han \& B. D. Reddy. On the finite element method for mixed variational inequalities arising in elastoplasticity. SIAM J. Numer. Anal, 32, 1778-1807, 1995.

[25] C. Carstensen, D. Peterseim \& M. Schedensack. Comparison results of finite element methods for the Poisson model problem. SIAM J. Numer. Anal, 50, No. 6, 2803-2823, 2012.

[26] R. Verfürth. Finite element appoximation of incompressible Navier-Stokes equations with slip boundary condition. Numer. Math, 50, 697-721, 1987.

[27] F. Brezzi \& M. Fortin. Mixed and Hybrid Finite Element Methods, Springer Series in Computational Mathematics, 15, 1991.

[28] P. Ciarlet. Finite Element Method for Elliptic Problems, North Holland, Amstedam, 1978.

[29] R. A. Adams. Sobolev Spaces, Academic Press, New York, 1975.

[30] V. Dolejsi \& M. Feistauer, and J. Felcman. On the discrete Friedrichs inequality for nonconforming finite elements. Numer. Funct. Anal. Optimiz., 20, 437-447, 1999.

[31] P. Knobloch. Uniform validity of discrete Friedrich's inequality for general nonconforming element spaces. Numer. Funct. Anal. Optimiz., 22, 107-126, 2001.

[32] S. Turek, A. Aouazzi, \& R. Schmachtel. Multigrid methods for stabilized nonconforming finite element for incompressible flow involving the deformation tensor formulation. J. Numer. Math., 10, No 3, 235-248, 2002.

[33] P. Grisvard. Elliptic Problems in Nonsmooth Domains, Pitman, 1985.

[34] R. Temam. Navier-Stokes equations: Theory and Numerical Analysis, 2nd ed., AMS Chelsea publishing, 2001.

[35] V. Girault \& B. Riviere \& M. Wheeler. A discontinuous galerkin method with nonoverlapping domain decomposition for the Stokes and Navier-Stokes problems. Math. Comp, 74,53-84, 2004.

[36] S. Brenner. Poincare-Friedrichs inequality for piecewise $H^{1}$ functions. SIAM J. Numer. Anal, 41, 306-324, 2003. 
[37] B.P. Lamichhane. A nonconforming finite element method for the Stokes equations using the Crouzeix-Raviart element for the velocity and the standard linear element for the pressure. Internat. J. Numer. Methods Fluids, 74, No 3, 222-228, 2014.

[38] S. C. Brenner. Two level additive Schwarz preconditioners for non conforming finite elements. In D. E. Keys and J. Xu, editors, Domain decomposition methods in scientific and engineering computing, AMS, Providence, 9-14, 1994.

[39] S. C. Brenner \& L. R. scott. The Mathematical Theory of Finite Element methods. Third Edition, Springer-Verlag, New-York, 2008.

[40] H. Brezis. Functional Analysis, Sobolev Spaces and partial differential equations. Springer, 2010.

[41] K. Atkinson \& W. Han. Theoretical Numerical Analysis: A Functional Analysis Framework, Texts in Applied Mathematics, vol 39, Second Edition, Springer, 2005. 